TITLE:

\title{
A new method for accurately determining the modal equivalent stiffness ratio of bonded piezoelectric structures
}

\section{$\operatorname{AUTHOR(S):~}$}

Yamada, Keisuke; Matsuhisa, Hiroshi; Utsuno, Hideo

\section{CITATION:}

Yamada, Keisuke ... [et al]. A new method for accurately determining the modal equivalent stiffness ratio of bonded piezoelectric structures. Journal of Sound and Vibration 2012 , 331(14): 3317-3344

ISSUE DATE:

2012-07

URL:

http://hdl.handle.net/2433/155457

\section{RIGHT:}

@ 2012 Elsevier Ltd.; この論文は出版社版でありません。引用の際には 出版社版をご確認ご利用ください。; This is not the published version. Please cite only the published version. 


\title{
A new method for accurately determining the modal equivalent stiffness ratio of bonded piezoelectric structures
}

\author{
Keisuke YAMADA* \\ Department of Mechanical Engineering and Science, Kyoto University, Yoshida-Honmachi, Sakyo-ku, Kyoto \\ 606-8501, Japan \\ Hiroshi MATSUHISA \\ Department of Mechanical Engineering and Science, Kyoto University, Yoshida-Honmachi, Sakyo-ku, Kyoto \\ 606-8501, Japan \\ Hideo UTSUNO \\ Department of Mechanical Engineering, Kansai University, 3-3-35 Yamate-cho, Suita-shi, Osaka 564-8680, \\ Japan
}

\begin{abstract}
This paper describes new methods for measuring the modal equivalent stiffness ratios and modal electromechanical coupling coefficients of piezoelectric elements attached to a host structure such as a beam. Modal equivalent stiffness ratios and modal electromechanical coupling coefficients are essential for estimating the performance and determining an optimum design of active vibration control and passive vibration suppression systems that use piezoelectric elements. Accurate determination of these modal parameters is also useful for other systems including piezoelectric sensors and energy generators. This paper not only describes the measurement methods but also presents the theoretical formulations derived by taking into account the effect of adhesive bonds. The formulations in this paper demonstrate the necessity of experimental measurements and the accuracy enhancements that the theoretical estimations can provide. Conventional methods for obtaining the modal equivalent stiffness ratios are sensitive to measurement errors, which result in the loss of accuracy, rendering these methods unreliable for many practical applications. The proposed methods use an inductor instead of an open circuit to address the abovementioned issue and, thereby, provide significant improvement in the accuracy. Because the loss factors of the experimental apparatus tend to compromise the accuracy of the proposed methods, a method using a negative resistor is proposed, theoretically analyzed, and confirmed to eliminate some of the errors introduced by loss factors. The advantages of the proposed methods and the effectiveness of theoretical analysis, considering the effect of adhesive bonds, are verified experimentally.
\end{abstract}

Key Words: Piezoelectric Element, Vibration Control, Flexible Structure, Smart Structure, Modal Analysis

*Corresponding author. Tel./fax: +81 757535195

E-mail address: yamada@me.kyoto-u.ac.jp (K. Yamada). 


\section{Introduction}

Vibration suppression methods using piezoelectric elements can be divided into two main categories: passive vibration suppression [1-5] and active vibration control [6, 7]. In addition, several hybrid vibration suppression methods have been studied in recent years [8-11]. Passive vibration suppression methods usually involve the use of either an inductive-resistive circuit or only a resistive circuit coupled to a piezoelectric element. The inductive -resistive circuit functions in a manner similar to that of a dynamic vibration absorber, and the resistive circuit functions as a Houde damper. If these circuits are optimally tuned, their performances will depend almost entirely on the equivalent stiffness ratio of the piezoelectric element [1]. Further, the optimum values of the circuit elements also depend on the equivalent stiffness ratio. On the other hand, the performance of active vibration control is determined on the basis of the electromechanical coupling coefficient of the piezoelectric element. This is because the control force is expressed as product of the electromechanical coupling coefficient and the voltage applied by the controller. Active methods use only the inverse piezoelectric effect, whereas passive methods use both the piezoelectric effect and the inverse piezoelectric effect. This is the reason why the equivalent stiffness ratio is related to the square of the electromechanical coupling coefficient. If either the equivalent stiffness ratio or the electromechanical coupling coefficient is known, the other can be determined. The mechanical and electrical energy conversion efficiency of the piezoelectric element attached to a host structure depends on the electromechanical coupling coefficient, and the value of the electromechanical coupling coefficient and the mode shape function of the host structure are closely related. If multiple vibration modes exist in the targeted frequency range, the modal electromechanical coupling coefficients for all the vibration modes must be obtained.

In prior literature, the equivalent stiffness ratio and the electromechanical coupling coefficient have been formulated theoretically under ideal conditions. Values of these parameters are derived on the basis of the properties of the piezoelectric material, sizes of the piezoelectric element and the host structure, and location of the piezoelectric element. However, in many cases these theoretical formulations do not agree well with the experimental ones. This disagreement occurs because of the inaccurate assumptions regarding the stiffness of the adhesive bonds under ideal conditions [1]. The restraint imposed by the adhesive bonds depends on their material properties, surface area, and thickness. The effect of adhesive bonds has not been investigated in detail with a viewpoint of vibration suppression because the experimentally measured values of equivalent stiffness ratio and electromechanical coupling coefficient that are used to study the effect of adhesive bonds are not sufficiently accurate. Similarly, the theoretically and experimentally obtained mode shapes are also different. In some cases, small differences in the mode shapes produce large differences in the performance of a system. In cases wherein the configuration of the host structure is complicated, it is difficult to obtain accurate mode shapes, even if numerical techniques such as the finite element method are employed.

In the research literature, the most widely used experimental method derives the equivalent stiffness ratio using the measured values of natural frequencies [2]. This method uses two natural frequencies corresponding to the short- and open-circuit states of the piezoelectric element. This method is simple and easy to implement in 
practical applications because it does not require the mode shape functions; however, it lacks accuracy when the difference between the two natural frequencies is small. In fact, in some cases, the equivalent stiffness ratio cannot be derived by any means using this method. In order to solve this problem, two new methods using an inductive circuit are proposed in this paper. We briefly described one of the methods [12]; the other method is based on the one proposed by Porfiri et al. [13]. The latter method proposed by us is more flexible than the one proposed by Porfiri et al. because it does not require precise tuning of inductance. The influence of loss factors such as the damping of the host structure on the equivalent stiffness ratio is investigated thoroughly in this study because, thus far, it has not been discussed in detail despite the fact that it cannot be neglected in practical applications.

In order to examine the accuracy of the theoretical estimations, governing equations are briefly derived by considering the effect of adhesive bonds on the basis of knowledge of bonding layers used for piezoelectric sensors [14-16]. The equivalent stiffness ratios are formulated using the conventional method as well as the proposed ones. The effects of frequency resolution, damping ratio of the system, dielectric loss in the piezoelectric element, and inductor loss on the accuracy of equivalent stiffness ratio are theoretically investigated. Because the losses in the piezoelectric element and the inductor reduce the accuracy of the equivalent stiffness ratio obtained by the proposed methods, a further improved method using a negative resistor is also presented. The effectiveness and advantages of the proposed methods are verified through simulations and experiments.

\section{Governing equations}

\subsection{Stiffness of adhesive bonds}

Piezoelectric elements generate voltage in response to the applied force and generate strain in response to the applied voltage; these phenomena are called the piezoelectric effect and inverse piezoelectric effect, respectively. There are several types of piezoelectric elements which are classified according to the directions of vibration, electrical field, and polarization. In this study, a plate-type piezoelectric element is used; however, there is no essential difference among the different types of piezoelectric elements that can be used with the proposed methods. Plate-type elements are often used for the suppression of bending vibrations because these elements have a low profile and are easy to work with. As shown in Fig. 1 (a), the vibrations and electrical field are produced in a direction perpendicular to each other. The plate-type piezoelectric element is attached to the host structure by using adhesive bonds, as shown in Fig. 1 (b). The bonding layer transmits force between the piezoelectric element and the host structure through shear coupling.

Analysis of the bonding layer is critical for studying its effect. The effect of the bonding layers has been investigated for piezoelectric sensors [14-16]. On the basis of this knowledge of bonding layers, the model shown in Fig. 2 (a) can be adopted as an approximate analytical model for the stiffness of the bonding layer. Here, for the sake of simplicity, the transverse stress applied to the piezoelectric element by the adhesive bond is assumed to be negligible, and $k_{a 1}$ is the total spring constant of the equivalent springs. The mechanical spring 
constant of the piezoelectric element in the longitudinal direction is expressed as

$$
k_{p 1}=E_{p} \frac{w_{p} t_{p}}{l_{p}},
$$

where $E_{p}$ is the Young's modulus of the piezoelectric element, and $l_{p}, w_{p}$, and $t_{p}$ are the length, width, and thickness of the piezoelectric element, respectively. From the knowledge of bonding layers, the spring constant ratio between $k_{a 1}$ and $k_{p 1}$ is expressed as follows [14]:

$$
\begin{aligned}
& r_{a 1}=\frac{k_{a 1}}{k_{p 1}}=\frac{1}{1+\frac{3 E_{p} w_{p} t_{p}}{E_{h} w_{h} t_{h}}}\left(\frac{\lambda l_{p}}{2} \frac{1}{\tanh \frac{\lambda l_{p}}{2}}-1\right), \\
& \lambda=\sqrt{\frac{G_{a}}{E_{p} t_{p} t_{a}}+\frac{3 w_{p} G_{a}}{E_{h} w_{h} t_{h} t_{a}}},
\end{aligned}
$$

where $\lambda$ is the shear lag parameter, $G_{a}$ is the transverse elasticity modulus of the adhesive bond, $t_{a}$ is the thickness of the bonding layer, $E_{h}$ is the Young's modulus of the host structure, and $w_{h}$ and $t_{h}$ are the width and thickness of the host structure, respectively.

The abovementioned formulations can be extended to obtain an approximate two-dimensional model [14]. For this purpose, the longitudinal and width directions of the one-dimensional model must be interchanged for obtaining the formulations of the width direction. Accordingly, we adopted the approximate analytical model shown in Fig. 2 (b), wherein the one-dimensional model is extended to develop a two-dimensional model. Here, $k_{a 2}$ is the total spring constant of the equivalent springs in the width direction. The deformation of the piezoelectric element is uniform in both the directions because the stress applied to the side surfaces of the piezoelectric element is uniform.

\subsection{Piezoelectric constitutive equations and equivalent mechanical models considering the stiffness of adhesive} bonds

The force equilibrium and the piezoelectric constitutive equations are given as

$$
\begin{gathered}
F_{1}=2 k_{a 1}\left(\frac{1}{2} x_{h}-\frac{1}{2} x_{p}\right), \\
F_{2}=2 k_{a 2}\left(\frac{1}{2} y_{h}-\frac{1}{2} y_{p}\right), \\
S_{1}=\frac{1}{E_{p}} T_{1}-v_{p} \frac{1}{E_{p}} T_{2}+d_{31} E_{3}, \\
S_{2}=-v_{p} \frac{1}{E_{p}} T_{1}+\frac{1}{E_{p}} T_{2}+d_{31} E_{3}, \\
D_{d 3}=d_{31} T_{1}+d_{31} T_{2}+\varepsilon_{33}^{T} E_{3},
\end{gathered}
$$

where $F_{1}$ and $F_{2}$ are the forces applied to the piezoelectric element by the equivalent springs in the longitudinal and width directions, respectively; $x_{p}$ and $y_{p}$ are the total displacement of the piezoelectric element in the longitudinal and width directions, respectively; and $x_{h}$ and $y_{h}$ are the total displacement of the 
connection points between the equivalent springs and the host structure in the longitudinal and width directions, respectively. Further, $S_{1}$ and $T_{1}$ are the strain and stress in the longitudinal direction, respectively; $S_{2}$ and $T_{2}$ are the strain and stress in the width direction, respectively; $E_{3}$ is the electrical field applied to the piezoelectric element; $D_{d 3}$ is the electrical displacement; $v_{p}$ is the Poisson's ratio of the piezoelectric element; $d_{31}$ is the piezoelectric constant; and $\varepsilon_{33}^{T}$ is the electrical permittivity. Subscripts 1,2 , and 3 denote the longitudinal, width, and thickness directions, respectively. Superscript $T$ denotes the value that is obtained under constant stress. We assumed that the piezoelectric element was isotropic in the longitudinal and width directions. In this research, a thin beam is used as the host structure, the beam and piezoelectric element are assumed to be aligned in the longitudinal direction. Yamada et al. derived an equivalent mechanical model using the same analytical model shown in Fig. 2 (b) [1]. Further, Yamada et al. assumed that $y_{h}=0$; however, this assumption can be adopted only if the beam is sufficiently wider than the piezoelectric element and if the piezoelectric element is placed around the center of the width direction. In this research, we adopted a more general condition wherein $x_{h}$ and $y_{h}$ are expressed as

$$
\begin{gathered}
x_{h}=S_{\text {hal }} l_{p}, \\
y_{h}=S_{\text {ha } 2} w_{p},
\end{gathered}
$$

where $S_{\text {hal }}$ and $S_{\text {ha2 }}$ are the average strains in the longitudinal and width directions, respectively, in the region of the host structure where the piezoelectric element is attached. Further, the average strain ratio of $S_{\text {hal }}$ to $S_{\text {ha2 }}$ is defined as

$$
b=-\frac{S_{\text {ha2 }}}{S_{\text {ha1 }}} .
$$

In general, $b$ is not equal to the Poisson's ratio of the host structure. From Eqs. (9)-(11), $y_{h}$ is expressed as

$$
y_{h}=-b \frac{w_{p}}{l_{p}} x_{h} .
$$

From Eqs. (4)-(8) and (12), the following equations are derived [1]:

$$
\begin{gathered}
x_{h}=\frac{1}{k_{p 1}^{\prime}} F_{1}+\frac{\theta_{p 1}^{\prime}}{k_{p 1}^{\prime}} V_{3}, \\
\frac{q}{1-b^{\prime}}=\theta_{p 1}^{\prime} x_{h}+\frac{C_{p}^{\prime S}}{1-b^{\prime}} V_{3}, \\
k_{p 1}^{\prime}=k_{p 1} \frac{1+\frac{1}{r_{a 2}}-b v_{p}}{\left(1+\frac{1}{r_{a 1}}\right)\left(1+\frac{1}{r_{a 2}}\right)-v_{p}^{2}}, \theta_{p 1}^{\prime}=\theta_{p 1} \frac{1+\frac{1}{r_{a 2}}+v_{p}}{\left(1+\frac{1}{r_{a 1}}\right)\left(1+\frac{1}{r_{a 2}}\right)-v_{p}^{2}}, \quad b^{\prime}=b \frac{1+\frac{1}{r_{a 1}}+v_{p}}{1+\frac{1}{r_{a 2}}+v_{p}}, \\
C_{p}^{\prime S}=C_{p}^{\prime T}-\frac{\theta_{p 1}^{\prime 2}}{k_{p 1}^{\prime}}\left(1-b^{\prime}\right), C_{p}^{\prime T}=C_{p}^{T}\left(1-\frac{d_{31}^{2} E_{p}}{\varepsilon_{33}^{T}} \frac{1+b}{1+\frac{1}{r_{a 2}}-b v_{p}}\right), C_{p}^{T}=\varepsilon_{33}^{T} \frac{l_{p} w_{p}}{t_{p}}, \\
\theta_{p 1}=d_{31} E_{p} w_{p}, r_{a 2}=\frac{k_{a 2}}{k_{p 2}}, k_{p 2}=E_{p} \frac{l_{p} t_{p}}{w_{p}}, V_{3}=E_{3} t_{p}, \quad q=D_{d 3} l_{p} w_{p} .
\end{gathered}
$$


From Eqs. (13) and (14), the equivalent mechanical model is developed, as shown in Fig. 3. Here, $\left(1-b^{\prime}\right) / \bar{C}_{p}^{S}$ is the spring constant, $\bar{\theta}_{p 1}^{\prime}$ is the length ratio of the virtual lever arms, $\bar{V}_{3}$ is the force, and $\bar{q} /\left(1-b^{\prime}\right)$ is the displacement. The virtual lever arms represent the internal electromechanical behavior of the model, instead of the physical elements. The geometry of the levers is determined by the sign of $\theta_{p 1}^{\prime}$. The levers do not exhibit inertial properties, and therefore, these properties are not considered. The values of $\bar{C}_{p}^{\prime S}, \bar{\theta}_{p 1}^{\prime}, \bar{V}_{3}$, and $\bar{q}$ are equal to those of $C_{p}^{\prime S}, \theta_{p 1}^{\prime}, V_{3}$, and $q$, respectively. The left half of the model, which consists of only a spring, represents the mechanical properties of the piezoelectric element, and the right half represents the electrical properties. The lever, thereby, provides the transducer's electromechanical coupling. The equivalent mechanical model can be reconstructed to obtain a model in which the function of a lever is integrated, as shown in Fig. 4. In this paper, the equivalent mechanical models shown in Figs. 3 and 4 are hereafter referred to as the equivalent mechanical models with and without a lever, respectively.

The spring constant of the electrical part is expressed as follows:

$$
\begin{gathered}
k_{e}^{\prime}=\left(1-b^{\prime}\right) \frac{\theta_{p 1}^{\prime 2}}{C_{p}^{\prime S}}=\left(1-b^{\prime}\right) \frac{\bar{\theta}_{p 1}^{\prime 2}}{\bar{C}_{p}^{\prime S}}=\frac{d_{31}^{\prime 2} E_{p}^{\prime}}{\frac{\varepsilon_{33}^{\prime T}}{1-b^{\prime}}-d_{31}^{\prime 2} E_{p}^{\prime}} k_{p 1}^{\prime}, \\
E_{p}^{\prime}=E_{p} \frac{1+\frac{1}{r_{a 2}}-b v_{p}}{\left(1+\frac{1}{r_{a 1}}\right)\left(1+\frac{1}{r_{a 2}}\right)-v_{p}^{2}}, d_{31}^{\prime}=d_{31} \frac{1+\frac{1}{r_{a 2}}+v_{p}}{1+\frac{1}{r_{a 2}}-b v_{p}}, \varepsilon_{33}^{\prime T}=\varepsilon_{33}^{T}\left(1-\frac{d_{31}^{2} E_{p}}{\varepsilon_{33}^{T}} \frac{1+b}{1+\frac{1}{r_{a 2}}-b v_{p}}\right) .
\end{gathered}
$$

In Eq. (27), $E_{p}^{\prime}$ is the apparent Young's modulus, and $E_{p}^{\prime}$ increases with an increase in $r_{a 1}$. On the other hand, as $r_{a 2}$ increases, both $E_{p}^{\prime}$ and $d_{31}^{\prime}$ increase, whereas $\varepsilon_{33}^{\prime T}$ decreases. Ratio $k_{e}^{\prime} / k_{p 1}^{\prime}$ increases with an increase in $r_{a 1}$ and $r_{a 2}$. These results imply that the efficiency of the electrical part of the piezoelectric element improves with an increase in $r_{a 1}$ and $r_{a 2}$.

\subsection{Governing equations for passive vibration suppression}

The analytical model consists of a cantilever, a piezoelectric element, and electrical impedance, as shown in Fig. 5. The cantilever is excited by an external force $f_{e}$. For simplicity, we assume that the piezoelectric element, owing to the effect of the adhesive bond, is rigidly attached to the host structure.

Flexible structures have an infinite number of bending vibration modes. Depending on a particular vibration mode, the attached piezoelectric element can be simultaneously subjected to tension and compression; this case is referred to as complex deformation. On the other hand, the case where the piezoelectric element is subjected to either tension or compression is referred to as simplex deformation. Because simplex deformation is a special case of complex deformation, complex deformation is investigated in this section.

As shown in Fig. 6, the piezoelectric element is divided into several parts depending on the deformation states. These parts are numbered as $1,2, \ldots, n$ from the clamped end side. The mechanical spring constant, the elemental electromechanical coupling coefficient, and the capacitances of the $\mathrm{j}$-th part are given as 


$$
k_{p 1}^{\prime j}=E_{p}^{\prime} \frac{w_{p} t_{p}}{l_{p}^{j}}, \quad \theta_{p 1}^{\prime j}=d_{31}^{\prime} E_{p}^{\prime} w_{p}=\theta_{p 1}^{\prime}, \quad C_{p}^{\prime T j}=\varepsilon_{33}^{\prime T} \frac{l_{p}^{j} w_{p}}{t_{p}}, C_{p}^{\prime S j}=C_{p}^{\prime T j}-\frac{\left(\theta_{p 1}^{\prime}\right)^{2}}{k_{p 1}^{\prime j}}\left(1-b^{\prime}\right),
$$

respectively, where $l_{p}^{j}$ is the length of the $\mathrm{j}$-th part with superscript $j$ denoting the $\mathrm{j}$-th part. The divided parts are electrically connected in parallel by the surface electrodes. The governing equations for passive vibration suppression can be obtained in a manner similar to that used by Yamada et al. [1]. We assume that the piezoelectric element is subjected to complex deformation in the i-th vibration mode. The equation of motion when the electrodes of the piezoelectric element are in a short-circuit state is expressed as

$$
\begin{gathered}
M_{i} \ddot{\xi}_{i}+D_{i} \dot{\xi}_{i}+K_{i} \xi_{i}=B_{i} f_{e} \\
M_{i}=M_{b i}+M_{p i}=1, \\
M_{b i}=\rho_{b} w_{b} t_{b} \int_{0}^{l_{b}} \psi_{i}^{2} \mathrm{~d} x \\
M_{p i}=\rho_{p} w_{p} t_{p} \int_{x_{L}}^{x_{R}} \psi_{i}^{2} \mathrm{~d} x \\
K_{i}=K_{b i}+K_{p i}, \\
K_{b i}=E_{b} w_{b}\left[\int_{0}^{x_{L}} \int_{-\frac{1}{2} t_{b}}^{\frac{1}{2} t_{b}} z^{2}\left(\frac{\partial^{2} \psi_{i}}{\partial x^{2}}\right)^{2} \mathrm{~d} z \mathrm{~d} x+\int_{x_{L}}^{x_{R}} \int_{-\left(t_{b}-t_{n}\right)}^{t_{n}} z^{2}\left(\frac{\partial^{2} \psi_{i}}{\partial x^{2}}\right)^{2} \mathrm{~d} z \mathrm{~d} x+\left.\int_{x_{R}}^{l_{b}} \int_{-\frac{1}{2} t_{b}}^{\frac{1}{2} t_{b}} z^{2}\left(\frac{\partial^{2} \psi_{i}}{\partial x^{2}}\right)^{2} \mathrm{~d} z \mathrm{~d} x\right|_{j},\right. \\
K_{p i}=\sum_{j=1}^{n} K_{p i}^{j}=E_{p}^{\prime} w_{p} \int_{x_{L}}^{x_{R}} \int_{t_{n}}^{t_{n}+t_{p}} z^{2}\left(\frac{\partial^{2} \psi_{i}}{\partial x^{2}}\right)^{2} \mathrm{~d} z \mathrm{~d} x \\
K_{p i}^{j}=E_{p}^{\prime} w_{p} \int_{x_{L}^{j}}^{x_{R}^{j}} \int_{t_{n}}^{t_{n}+t_{p}} z^{2}\left(\frac{\partial^{2} \psi_{i}}{\partial x^{2}}\right)^{2} \mathrm{~d} z \mathrm{~d} x, \\
D_{i}=2 \gamma_{i} \sqrt{M_{i} K_{i}}, \\
B_{i}=\psi_{i}\left(x_{f}\right) \\
E_{b} w_{b} t_{b}^{2}-E_{p}^{\prime} w_{p} t_{p}^{2} \\
2\left(E_{b} w_{b} t_{b}+E_{p}^{\prime} w_{p} t_{p}\right)
\end{gathered}
$$

where $\xi_{i}$ is the modal displacement; $M_{i}$ is the modal mass; $D_{i}$ is the modal damping coefficient; $K_{i}$ is the modal stiffness; $B_{i}$ is the modal influence coefficient of the external force; $\gamma_{i}$ is the modal damping ratio; $\rho_{b}$ and $\rho_{p}$ are the densities of the beam and the piezoelectric element, respectively; $E_{b}$ is the Young's modulus of the beam; $l_{b}, w_{b}$, and $t_{b}$ are the length, width, and thickness of the beam, respectively; and $t_{n}$ is the distance between the neutral axis and the upper side of the beam in the region where the piezoelectric element is attached. Further, $x_{L}$ and $x_{R}$ are the distances between the clamped end and the left and right endpoints of the piezoelectric element, respectively, and $x_{f}$ is the distance between the clamped end and the point at which the external force is applied. $x_{L}^{j}$ and $x_{R}^{j}$ are the distances between the clamped end and the left and right endpoints of the $\mathrm{j}$-th part, respectively, and $\psi_{i}$ is the shape function of the vibration mode. The $x$ axis represents the longitudinal direction, and the clamped end represents the origin. Variable $z$ denotes the distance from the neutral axis. Subscript $i$ denotes the $\mathrm{i}$-th vibration mode. Because the cantilever in the analytical model is thin, the shear deformation and rotary inertia of the cantilever are ignored. As shown in Eq. (35), the shape 
function $\psi_{i}$ is normalized with respect to the modal mass $M_{i}$. When the electrodes are shorted, the electrical properties of the piezoelectric element do not affect the vibration system. Because $K_{p i}^{j}$ is derived from $k_{p 1}^{\prime j}$, the modal influence coefficient of the $\mathrm{j}$-th part $\theta_{k i}^{j}$ is expressed as

$$
\theta_{k i}^{j}=\operatorname{sgn}\left(\xi_{i} x_{h}^{j}\right) \sqrt{\frac{K_{p i}^{j}}{k_{p 1}^{\prime j}}},
$$

where $x_{h}^{j}$ is the displacement of the $\mathrm{j}$-th part in the longitudinal direction. The influence of the $\mathrm{j}$-th part on the main system is determined by $\theta_{k i}^{j}$. Figure 7 (a) shows the equivalent mechanical model with levers for the targeted vibration mode when the electrodes of the piezoelectric element are shunted with the electrical impedance $Z_{e}$. In this model, $q^{j}$ is the charge of the $\mathrm{j}$-th part, and $\bar{Z}_{e}$ and $\bar{q}^{j} /\left(1-b^{\prime}\right)$ are the damping coefficient and displacement, respectively. The values of $\bar{Z}_{e}$ and $\bar{q}^{j}$ are equal to those of $Z_{e}$ and $q^{j}$, respectively. The mechanical element for the electrical impedance is omitted and only the case wherein both $\theta_{p 1}^{\prime}$ and $\theta_{k i}^{j}$ are positive is shown in the figure. The equivalent mechanical model shown in Fig. 7 (a) provides the following objective governing equations:

$$
\begin{gathered}
M_{i} \ddot{\xi}_{i}+D_{i} \dot{\xi}_{i}+K_{i} \xi_{i}+\sum_{j=1}^{n} \Theta_{i}^{j}\left[\left(1-b^{\prime}\right) \frac{\Theta_{i}^{j}}{C_{p}^{\prime S j}} \xi_{i}-\left.\frac{1}{C_{p}^{\prime S j}} q^{j}\right|_{]}=B_{i} f_{e},\right. \\
Z_{e} \dot{q}=\left(1-b^{\prime}\right) \frac{\Theta_{i}^{1}}{C_{p}^{\prime S 1}} \xi_{i}-\frac{1}{C_{p}^{S 1}} q^{1}=\cdots=\left(1-b^{\prime}\right) \frac{\Theta_{i}^{j}}{C_{p}^{\prime S j}} \xi_{i}-\frac{1}{C_{p}^{\prime S j}} q^{j}=\cdots=\left(1-b^{\prime}\right) \frac{\Theta_{i}^{n}}{C_{p}^{\prime S n}} \xi_{i}-\frac{1}{C_{p}^{\prime S n}} q^{n}, \\
\Theta_{i}^{j}=\theta_{p 1}^{\prime} \theta_{k i}^{j}, \quad q=\sum_{j=1}^{n} q^{j} .
\end{gathered}
$$

Equations (46) and (47) can be simplified as

$$
\begin{gathered}
M_{i} \ddot{\xi}_{i}+D_{i} \dot{\xi}_{i}+K_{i} \xi_{i}+\Theta_{i}\left[\left(1-b^{\prime}\right) \frac{\Theta_{i}}{C_{p}^{\prime S}} \xi_{i}-\left.\frac{1}{C_{p}^{\prime S}} q\right|_{]}=B_{i} f_{e},\right. \\
Z_{e} \dot{q}=\left(1-b^{\prime}\right) \frac{\Theta_{i}}{C_{p}^{\prime}} \xi_{i}-\frac{1}{C_{p}^{\prime S}} q,
\end{gathered}
$$

where $\Theta_{i}$ is the modal electromechanical coupling coefficient expressed as

$$
\Theta_{i}=\sum_{j=1}^{n} \Theta_{i}^{j}=\theta_{p 1}^{\prime} \theta_{k i}, \quad \theta_{k i}=\sum_{j=1}^{n} \theta_{k i}^{j},
$$

where $\theta_{k i}$ is the modal influence coefficient of the piezoelectric element. The signs of $\theta_{k i}^{j}$ in the regions of tension and compression are different, and therefore, $\theta_{k i}^{j}$ in the compression and tension regions cancel each other because the signs of the charges generated in these regions are different. The fourth term on the left-hand side of Eq. (50) denotes the force resulting from the inverse piezoelectric effect, whereas the first term on the right-hand side of Eq. (51) denotes the voltage generated by the piezoelectric effect. Because both the effects contribute to passive vibration suppression, the system performance can be evaluated by the modal equivalent stiffness ratio defined as

$$
\beta_{i}=\left(1-b^{\prime}\right) \frac{\Theta_{i}^{2}}{K_{i} C_{p}^{\prime S}}
$$

The square root of the modal equivalent stiffness ratio is referred to as the nondimensional electromechanical 
coupling coefficient or the generalized electromechanical coupling coefficient. On the basis of Eqs. (50) and (51), a simplified equivalent mechanical model without levers is shown in Fig. 7 (b). The equivalent stiffness ratio corresponds to the ratio of the spring constants in the equivalent mechanical model. The value of the modal equivalent stiffness ratio is certainly less than 1 .

\subsection{Governing equation for active vibration control}

To obtain formulations for active vibration control, a control voltage is required to be applied to the piezoelectric element instead of adding electrical impedance to the element. Vibration of the host structure is actively controlled by using the piezoelectric element as an actuator. The equation of motion is given as follows:

$$
M_{i} \ddot{\xi}_{i}+D_{i} \dot{\xi}_{i}+K_{i} \xi_{i}+\Theta_{i} V_{A}=B_{i} f_{e},
$$

where $V_{A}$ is the control voltage. The voltage applied by electrical impedance in Eq. (50) is replaced by the control voltage. If the value of the modal equivalent stiffness ratio is known, the modal electromechanical coupling coefficient can be derived as follows:

$$
\Theta_{i}= \pm \sqrt{\frac{\beta_{i} K_{i} C_{p}^{\prime S}}{1-b^{\prime}}} .
$$

The sign of the modal electromechanical coupling coefficient can be determined from the polarity of the electrodes and the shape function of the vibration mode.

\section{Methods to derive modal equivalent stiffness ratio}

In this section, we describe the two methods proposed for deriving the modal equivalent stiffness ratio experimentally. In the preceding section, the dielectric loss in the piezoelectric element was ignored; however, it affects the accuracy of the derived parameters. Hence, the equivalent circuit of a piezoelectric element with the dielectric loss, as shown in Fig. 8, is used in the following analysis. The equivalent parallel resistance $R_{p}$ is defined as

$$
R_{p}=\frac{1}{\omega C_{p}^{\prime S} \tan \delta_{p}},
$$

where $\omega$ is the excitation angular frequency, and $\tan \delta_{p}$ is the dielectric loss tangent of the piezoelectric element attached to the host structure. When $\tan \delta_{p}=0$, no energy loss occurs in the piezoelectric element.

\subsection{Conventional method}

The conventional method used for experimentally determining the modal equivalent stiffness ratio requires measurement of natural frequencies when the electrodes are short- and open-circuited [2]. When the electrodes of the piezoelectric element are shorted, the governing equation is simplified as

$$
M_{i} \ddot{\xi}_{i}+D_{i} \dot{\xi}_{i}+K_{i} \xi_{i}=B_{i} f_{e} .
$$

From Eq. (58), the nondimensional compliance is obtained as 


$$
\begin{gathered}
\frac{\Xi_{i}}{\Xi_{\mathrm{st} i}}=\frac{1}{-g_{i}^{2}+2 \mathrm{j} \gamma_{i} g_{i}+1}, \\
\Xi_{\mathrm{st} i}=\frac{B_{i} F_{e}}{K_{i}}, g_{i}=\frac{\omega}{\Omega_{i}}, \Omega_{i}=\sqrt{\frac{K_{i}}{M_{i}}},
\end{gathered}
$$

where $\Xi_{i}$ is the complex amplitude of $\xi_{i}, F_{e}$ is the amplitude of $f_{e}$, and $\mathrm{j}$ is the imaginary unit. From Eq. (59), the nondimensional undamped natural frequency with shorted electrodes is derived as

$$
g_{S}=1 .
$$

In contrast, when the piezoelectric element is in an open-circuit state, the circuit equation is expressed as

$$
R_{p} \dot{q}=\left(1-b^{\prime}\right) \frac{\Theta_{i}}{C_{p}^{\prime S}} \xi_{i}-\frac{1}{C_{p}^{\prime S}} q
$$

From Eqs. (50), (57), and (64), the nondimensional compliance is obtained as

$$
\frac{\Xi_{i}}{\Xi_{\mathrm{st} i}}=\frac{1}{-g_{i}^{2}+2 \mathrm{j} \gamma_{i} g_{i}+1+\beta_{i} \frac{\mathrm{j}}{\tan \delta_{p}+\mathrm{j}}} .
$$

Under an ideal condition of $\tan \delta_{p}=0$, the nondimensional undamped natural frequency for the piezoelectric element in an open-circuit state is obtained as

$$
g_{O}=\sqrt{1+\beta_{i}} .
$$

From Eqs. (63) and (66), the modal equivalent stiffness ratio is formulated as

$$
\begin{gathered}
\beta_{i}=g_{O}^{2}-1=\frac{\Omega_{O}^{2}}{\Omega_{S}^{2}}-1, \\
\Omega_{S}=\Omega_{i}, \Omega_{O}=\Omega_{i} \sqrt{1+\beta_{i}},
\end{gathered}
$$

where $\Omega_{S}$ and $\Omega_{O}$ are the undamped natural angular frequencies for short- and open-circuited electrodes, respectively. It should be noted that Eq. (67) requires only the natural frequencies. The shape function of the targeted vibration mode is not required.

The natural frequencies can be determined experimentally by different methods. The Bode plot of compliance is used in this research because it is the most popular approach for obtaining the frequency response of a system. The frequency at which a peak occurs in the magnitude plot of the compliance is considered as the natural frequency in the experiment, for simplicity. However, in some cases, the modal equivalent stiffness ratio obtained using this method may be inaccurate because this method provides damped natural frequencies instead of undamped ones. The effect of the modal damping ratio $\gamma_{i}$ and the dielectric loss tangent $\tan \delta_{p}$ on the accuracy of the modal equivalent ratio is investigated in section 4. Mobility and accelerance can also be used instead of compliance to determine the natural frequencies; however, we have used compliance as an example in the following sections.

\subsection{Two-peak method}

The analytical model of the two-peak method is shown in Fig. 9. An inductor is coupled to the piezoelectric element. Because the model shown in Fig. 9 has two degrees of freedom in the frequency range with respect to 
the i-th vibration mode, two resonance peaks appear in the frequency response function. The inductor should be tuned in order achieve two peaks of almost equal amplitude. The details required for optimum tuning of the inductor are described in the following subsection. Further, any type of inductor can be used with this method; however, an inductor simulated using the generalized impedance converter is used in this study because of the ease with which it can be tuned. The schematic diagram of the simulated inductor is shown in Fig. 10 (a). The nominal inductance $L_{g}$ is defined as

$$
L_{g}=\frac{R_{1} R_{3} C_{4} R_{5}}{R_{2}},
$$

where $R_{1}, R_{2}, R_{3}$, and $R_{5}$ are the resistances in the model, and $C_{4}$ is the capacitance. A variable resistor is used to tune the inductance. The dielectric loss of the capacitor is also taken into consideration to investigate its effect. The equivalent parallel resistance of the capacitor is given as

$$
R_{4}=\frac{1}{\omega C_{4} \tan \delta_{c}},
$$

where $\tan \delta_{c}$ is the dielectric loss tangent of the capacitor. Because the equivalent circuit of the capacitor is represented by a parallel connection of $C_{4}$ and $R_{4}$, the exact electrical impedance of the simulated inductor is obtained as

$$
\begin{aligned}
& Z_{g}=\mathrm{j} \omega L_{g}+R_{g}, \\
& R_{g}=\omega L_{g} \tan \delta_{c},
\end{aligned}
$$

where $R_{g}$ is the resistance of the simulated inductor. The equivalent circuit of the simulated inductor is depicted in Fig. 10 (b). The circuit equation of the model shown in Fig. 9 is written as

$$
L_{g} \ddot{q}_{2}+R_{g} \dot{q}_{2}=R_{p}\left(\dot{q}-\dot{q}_{2}\right)=\left(1-b^{\prime}\right) \frac{\Theta_{i}}{C_{p}^{\prime S}} \xi_{i}-\frac{1}{C_{p}^{\prime S}} q,
$$

where $q_{2}$ is the charge that passes through the inductor. Using Eqs. (50), (57), and (74), we derive the nondimensional compliance as

$$
\begin{gathered}
\frac{\Xi_{i}}{\Xi_{\mathrm{st} i}}=\frac{1}{-g_{i}^{2}+2 \mathrm{j} \gamma_{i} g_{i}+1+\beta_{i} \frac{-g_{i}^{2}+\mathrm{j} \tan \delta_{c} \cdot g_{i}^{2}}{\left(-1+\tan \delta_{c} \tan \delta_{p}\right) g_{i}^{2}+\mathrm{j}\left(\tan \delta_{c}+\tan \delta_{p}\right) g_{i}^{2}+f_{i}^{2}}}, \\
f_{i}=\frac{\omega_{a}}{\Omega_{i}}, \omega_{a}=\sqrt{\frac{1}{L_{g} C_{p}^{\prime S}}} .
\end{gathered}
$$

For the cases in which the loss terms are negligible, Eq. (75) is simplified as

$$
\frac{\Xi_{i}}{\Xi_{\mathrm{st} i}}=\frac{1}{-g_{i}^{2}+1+\beta_{i} \frac{-g_{i}^{2}}{-g_{i}^{2}+f_{i}^{2}}} .
$$

From Eq. (78), the two nondimensional undamped resonance frequencies are obtained as

$$
g_{L, R}=\sqrt{\frac{1+f_{i}^{2}+\beta_{i} \mp \sqrt{\left(1+f_{i}^{2}+\beta_{i}\right)^{2}-4 f_{i}^{2}}}{2}} .
$$

Using $g_{L}$ and $g_{R}$, we derive the modal equivalent stiffness ratio as 


$$
\beta_{i}=\left(1-g_{L}^{2}\right)\left(g_{R}^{2}-1\right)=\left(1-\frac{\Omega_{L}^{2}}{\Omega_{S}^{2}}\right)\left(\frac{\Omega_{R}^{2}}{\Omega_{S}^{2}}-1\right),
$$

where $\Omega_{L}$ and $\Omega_{R}$ are the undamped angular resonance frequencies. It should be noted that Eq. (80) requires only three frequencies and does not include the natural frequency ratio $f_{i}$. The derived equation indicates that the value of the inductance is not required when using this method. The effects of the modal damping ratio and the dielectric loss tangents on the equivalent stiffness ratio obtained using the proposed methods as well as the conventional method are investigated in section 4 .

\subsection{Two-point intersection method}

The analytical model of the two-point intersection method is shown in Fig. 11. The resistances of the piezoelectric element and the simulated inductor are represented separately in this model. Further, a resistor is added to the model shown in Fig. 9.

The circuit equation is given as

$$
L_{g} \ddot{q}_{2}+R_{g} \dot{q}_{2}=\frac{R_{p} R_{A}}{R_{p}+R_{A}}\left(\dot{q}-\dot{q}_{2}\right)=\left(1-b^{\prime}\right) \frac{\Theta_{i}}{C_{p}^{\prime S}} \xi_{i}-\frac{1}{C_{p}^{\prime S}} q,
$$

where $R_{A}$ is the resistance of the additional resistor. From Eqs. (50), (57), and (81), the nondimensional compliance is obtained as

$$
\begin{gathered}
\frac{\Xi_{i}}{\Xi_{\mathrm{st} i}}=\frac{1}{-g_{i}^{2}+2 \mathrm{j} \gamma_{i} g_{i}+1+\beta_{i} \frac{-g_{i}^{2}+\mathrm{j} \tan \delta_{c} \cdot g_{i}^{2}}{\left(-1+\tan \delta_{c} \tan \delta_{p}\right) g_{i}^{2}+\mathrm{j}\left(\tan \delta_{c}+\tan \delta_{p}\right) g_{i}^{2}+2 \mathrm{j} \zeta_{A} f_{i} g_{i}+f_{i}^{2}+2 \tan \delta_{c} \cdot \zeta_{A} f_{i} g_{i}}}, \\
\zeta_{A}=\frac{1}{2 R_{A}} \sqrt{\frac{L_{g}}{C_{p}^{\prime S}}} .
\end{gathered}
$$

In the case when $\gamma_{i}$ is negligible, the magnitude of the nondimensional compliance (82) has two fixed points that are independent of $\zeta_{A}$ and $\tan \delta_{p}$. The two fixed nondimensional frequencies are derived as

$$
g_{P, Q}=\sqrt{\frac{1+\frac{f_{i}^{2}}{1+\tan ^{2} \delta_{c}}+\frac{1}{2} \beta_{i} \mp \sqrt{\left(1+\frac{f_{i}^{2}}{1+\tan ^{2} \delta_{c}}+\frac{1}{2} \beta_{i}\right)^{2}-4 \frac{f_{i}^{2}}{1+\tan ^{2} \delta_{c}}}}{2}} .
$$

The modal equivalent stiffness ratio is defined using $g_{P}$ and $g_{Q}$ as follows:

$$
\beta_{i}=2\left(1-g_{P}^{2}\right)\left(g_{Q}^{2}-1\right)=2\left(1-\frac{\Omega_{P}^{2}}{\Omega_{S}^{2}}\right)\left(\frac{\Omega_{Q}^{2}}{\Omega_{S}^{2}}-1\right),
$$

where $\Omega_{P}$ and $\Omega_{Q}$ are the angular frequencies of the two fixed points. In the experiment, the two fixed points are obtained by varying the value of $R_{A}$. The intersection points of the magnitude plots using $R_{A}=0$ and $R_{A}=\infty$ are obtained easily because they correspond to the short-circuit condition and a condition in which only the inductor is considered, respectively. $R_{A}=\infty$ is employed in the following. When $R_{A}=\infty$ is substituted in Eq. (83), Eq. (82) is simplified to Eq. (75). The effect of the loss factors on the accuracy of the 
derived modal equivalent stiffness ratio is investigated in section 4.

\subsection{Measurement error tolerance in equivalent stiffness ratio and optimum frequency ratio}

The frequencies obtained by performing experiments include measurement error. This error reduces the accuracy of the modal equivalent stiffness ratio. We assume that $\hat{g}_{O}^{2}, \hat{g}_{L}^{2}, \hat{g}_{R}^{2}, \hat{g}_{P}^{2}$, and $\hat{g}_{Q}^{2}$ are the measured values. These measured values can be considered to consist of true values and errors as follows:

$$
\hat{g}_{O}^{2}=g_{O}^{2}+\varepsilon_{O}, \quad \hat{g}_{L}^{2}=g_{L}^{2}+\varepsilon_{L}, \quad \hat{g}_{R}^{2}=g_{R}^{2}+\varepsilon_{R}, \quad \hat{g}_{P}^{2}=g_{P}^{2}+\varepsilon_{P}, \quad \hat{g}_{Q}^{2}=g_{Q}^{2}+\varepsilon_{Q},
$$

where the circumflex ^ denotes the measured values. Further, the first and second terms on the right-hand sides of the expressions represent the true values and errors, respectively. We assume that the errors are random and that their absolute values do not differ significantly. The modal equivalent stiffness ratio with error is obtained as

$$
\hat{\beta}_{i}=\left\{\begin{array}{ll}
\left(g_{O}^{2}-1\right)+\varepsilon_{O} & \text { (Conventional method) } \\
\left(1-g_{L}^{2}\right)\left(g_{R}^{2}-1\right)+\left[\varepsilon_{R}\left(1-g_{L}^{2}\right)-\varepsilon_{L}\left(g_{R}^{2}-1\right)-\varepsilon_{L} \varepsilon_{R}\right] & \text { (Two-peak method) } \\
2\left(1-g_{P}^{2}\right)\left(g_{Q}^{2}-1\right)+\left[2 \varepsilon_{Q}\left(1-g_{P}^{2}\right)-2 \varepsilon_{P}\left(g_{Q}^{2}-1\right)-2 \varepsilon_{P} \varepsilon_{Q}\right] & \text { (Two-point intersection method) }
\end{array},\right.
$$

where the first terms for all the methods correspond to the true values of $\beta_{i}$, and the remaining terms correspond to the errors. The condition that minimizes the expected values of the error is given as

$$
\begin{cases}g_{R}^{2}-1=1-g_{L}^{2} & \text { (Two-peak method) } \\ g_{Q}^{2}-1=1-g_{P}^{2} & \text { (Two-point intersection method })\end{cases}
$$

where $\varepsilon_{L} \varepsilon_{R}$ and $\varepsilon_{P} \varepsilon_{Q}$ are ignored because they are significantly small. Using Eqs. (79), (84), and (92), we derive the optimum frequency ratio as

$$
f_{\text {opti }}=\left\{\begin{array}{ll}
\sqrt{1-\beta_{i}} & \text { (Two-peak method) } \\
\sqrt{\left(1+\tan ^{2} \delta_{c}\right)\left(1-\frac{1}{2} \beta_{i}\right)} & \text { (Two-point intersection method) }
\end{array} .\right.
$$

The optimum frequency ratio $f_{\mathrm{opt} i}$ is used in the following. Substituting Eq. (93) in Eqs. (79) and (84), we obtain $g_{L}, g_{R}, g_{P}$, and $g_{Q}$ as

$$
\begin{gathered}
g_{L, R}=\sqrt{1 \mp \sqrt{\beta_{i}}}, \\
g_{P, Q}=\sqrt{1 \mp \sqrt{\frac{1}{2} \beta_{i}}} .
\end{gathered}
$$

Substitution of Eqs. (94) and (95) in Eq. (91) gives

$$
\hat{\beta}_{i}=\left\{\begin{array}{ll}
\beta_{i}+\varepsilon_{O} & \text { (Conventional method) } \\
\beta_{i}+\left[\sqrt{\beta_{i}}\left(\varepsilon_{R}-\varepsilon_{L}\right)-\varepsilon_{L} \varepsilon_{R}\right] & \text { (Two-peak method) } \\
\beta_{i}+\left[\sqrt{2 \beta_{i}}\left(\varepsilon_{Q}-\varepsilon_{P}\right)-2 \varepsilon_{P} \varepsilon_{Q}\right] & \text { (Two-point intersection method) }
\end{array} .\right.
$$

From the definition of $\beta_{i}$, it is known that the value of $\beta_{i}$ is less than 1 ; therefore, the measurement errors introduced by the two proposed methods are lower than those introduced by the conventional method. This is because the true value and the error are independent of each other in the expression of $\hat{\beta}_{i}$ for the conventional 
method; thus, the accuracy of $\hat{\beta}_{i}$ is compromised when $\varepsilon_{O}$ is not negligible compared with $\beta_{i}$. In contrast, the errors introduced by the proposed methods depend on $\beta_{i}$. Therefore, the value of $\hat{\beta}_{i}$ obtained by the proposed methods is accurate even if the value of $\beta_{i}$ is mall, because the errors are dependent on the value of $\beta_{i}$. Equation (96) indicates that the $\hat{\beta}_{i}$ obtained by the two-peak method is slightly more accurate than that obtained by the two-point intersection method. However, the two-point intersection method has an advantage over the two-peak method, i.e., in the two-point intersection method, the accuracy of $\hat{\beta}_{i}$ is not affected by the presence of $\tan \delta_{c}$ and $\tan \delta_{p}$ under the condition when the modal damping ratio can be ignored.

\subsection{Quantization of modal equivalent stiffness ratio by frequency resolution}

The conventional and proposed methods require measurement of a few frequencies. The frequencies that are obtained by performing experiments have a certain frequency resolution. The frequency resolution causes quantization of the measured modal equivalent stiffness ratio. Using the angular frequency resolution $\Delta \omega$, we find that the measured angular frequencies are given as

$$
\hat{\Omega}_{S}=n_{S} \Delta \omega, \hat{\Omega}_{O}=n_{O} \Delta \omega, \hat{\Omega}_{L}=n_{L} \Delta \omega, \hat{\Omega}_{R}=n_{R} \Delta \omega, \hat{\Omega}_{P}=n_{P} \Delta \omega, \hat{\Omega}_{Q}=n_{Q} \Delta \omega
$$

where $n_{S}, n_{O}, n_{L}, n_{R}, n_{P}$, and $n_{Q}$ are the numbers of $\Delta \omega$ included in $\hat{\Omega}_{S}, \hat{\Omega}_{O}, \hat{\Omega}_{L}, \hat{\Omega}_{R}, \hat{\Omega}_{P}$, and $\hat{\Omega}_{Q}$, respectively. The measured modal equivalent stiffness ratio is expressed as

$$
\begin{aligned}
& \left(\frac{n_{O}}{n_{S}}\right)^{2}-1 \quad \text { (Conventional method) } \\
& \hat{\beta}_{i}=\left\{\begin{array}{l}
{\left[1-\left.\left(\frac{n_{L}}{n_{S}}\right)^{2}\right|_{\rfloor}\left[\left(\frac{n_{R}}{n_{S}}\right)^{2}-\left.1\right|_{\rfloor} \quad\right. \text { (Two-peak method) }\right.} \\
2\left[1-\left.\left(\frac{n_{P}}{n_{S}}\right)^{2}\right|_{\mid}\right]\left[\left(\frac{n_{Q}}{n_{S}}\right)^{2}-\left.1\right|_{\rfloor}\right. \text {(Two-point intersection method) }
\end{array}\right.
\end{aligned}
$$

In general, the value of the modal equivalent stiffness ratio is significantly lower than 1. Under this condition, Eq. (103) can be approximately expressed as follows:

$$
\hat{\beta}_{i} \approx\left\{\begin{array}{ll}
2\left(\frac{n_{O}}{n_{S}}-1\right) & \text { (Conventional method) } \\
4\left(1-\frac{n_{L}}{n_{S}}\right)\left(\frac{n_{R}}{n_{S}}-1\right) & \text { (Two-peak method) } \\
8\left(1-\frac{n_{P}}{n_{S}}\right)\left(\frac{n_{Q}}{n_{S}}-1\right) & \text { (Two-point intersection method) }
\end{array} .\right.
$$

Further, on the basis of Eqs. (94) and (95), the following relations are formulated:

$$
1-\frac{n_{L}}{n_{S}} \approx \frac{\sqrt{\beta_{i}}}{2}, \frac{n_{R}}{n_{S}}-1 \approx \frac{\sqrt{\beta_{i}}}{2}, 1-\frac{n_{P}}{n_{S}} \approx \frac{1}{2} \sqrt{\frac{\beta_{i}}{2}}, \frac{n_{Q}}{n_{S}}-1 \approx \frac{1}{2} \sqrt{\frac{\beta_{i}}{2}} .
$$

From Eqs. (104)-(108), the approximate resolution of the measured modal equivalent stiffness ratio $\hat{\beta}_{i}$ is 
defined as

$$
\Delta \hat{\beta}_{i} \approx\left\{\begin{array}{ll}
\frac{2}{n_{S}} & \text { (Conventional method) } \\
\frac{2}{n_{S}} \sqrt{\beta_{i}} & \text { (Two-peak method) } \\
\frac{2}{n_{S}} \sqrt{2 \beta_{i}} & \text { (Two-point intersection method) }
\end{array} .\right.
$$

The ratio of $\beta_{i}$ to $\Delta \hat{\beta}_{i}$ is critical in evaluating the accuracy of $\hat{\beta}_{i}$. However, it is impossible to obtain the true value of $\beta_{i}$ in principle. Therefore, a resolution evaluation index is defined using $\hat{\beta}_{i}$ instead of $\beta_{i}$, as follows:

$$
A_{r}=\frac{\hat{\beta}_{i}}{\Delta \hat{\beta}_{i} \approx}\left\{\begin{array}{ll}
\frac{n_{S}}{2} \hat{\beta}_{i}=n_{O}-n_{S} & \text { (Conventional method) } \\
\frac{n_{S}}{2} \sqrt{\hat{\beta}_{i}}=\sqrt{\left(n_{S}-n_{L}\right)\left(n_{R}-n_{S}\right)} & \text { (Two-peak method) } \\
\frac{n_{S}}{2} \sqrt{\frac{\hat{\beta}_{i}}{2}}=\sqrt{\left(n_{S}-n_{P}\right)\left(n_{Q}-n_{S}\right)} & \text { (Two-point intersection method) }
\end{array} .\right.
$$

The larger the value of the resolution evaluation index $A_{r}$, the higher is the accuracy of $\hat{\beta}_{i}$. Equation (110) indicates that values of $A_{r}$ for the proposed methods are better than the value obtained for the the conventional one.

\section{Effect of modal damping ratio and dielectric loss tangents}

The modal equivalent stiffness ratio is derived under the assumption that all or some of the loss factors are negligible. However, for the cases in which the loss factors are not negligible, the accuracy of the estimated modal equivalent stiffness ratio reduces. In subsection 3.4, the proposed methods were found to have higher tolerance toward the measurement errors; however, reduction in accuracy is inevitable if the measurement errors are large. In this study, we assume that the frequencies are obtained using the magnitude plot of the measured compliances. In this case, the natural angular frequency, when the piezoelectric element is in the short-circuit state, is also affected by the modal damping ratio.

$$
\Omega_{S}^{d}=\Omega_{S} \sqrt{1+2 \gamma_{i}^{2}},
$$

where $\Omega_{S}^{d}$ is the so-called damped natural angular frequency.

In addition, the effect of the modal damping ratio and the dielectric loss tangents on the accuracy of the modal equivalent stiffness ratio depends on the value of the modal equivalent stiffness ratio itself. Equation (96) indicates that the loss factors largely affect the accuracy if the value of the modal equivalent stiffness ratio is small. In order to investigate this effect, the normalized nondimensional compliances are used. The significance of this effect is highlighted by considering that the value of the modal equivalent stiffness ratio is significantly smaller than 1 . 


\subsection{Normalization of nondimensional compliance}

In order to normalize the nondimensional compliance expressed by Eq. (65) with respect to the conventional method, the following parameters are introduced:

$$
\bar{u}_{O i}=\frac{\frac{\Xi_{i}}{\Xi_{\mathrm{sti}}}}{\frac{2}{\beta_{i}}}, \bar{G}_{O i}=\frac{g_{i}^{2}-1}{\beta_{i}}, \bar{\gamma}_{O i}=\frac{\gamma_{i}}{\frac{\beta_{i}}{4}} .
$$

Substitution of Eqs. (112)-(114) in Eq. (65) gives the normalized nondimensional compliance for the open-circuit state as follows:

$$
\bar{u}_{O i} \approx \frac{1}{2} \frac{1}{-\bar{G}_{O i}+\frac{1}{2} \mathrm{j} \bar{\gamma}_{O i}+\frac{\mathrm{j}}{\tan \delta_{p}+\mathrm{j}}},
$$

where $1+\beta_{i} \bar{G}_{O i} \approx 1$ is assumed because $\beta_{i}<<1$. It can be observed that the normalized nondimensional compliance (115) does not include $\beta_{i}$. Therefore, the effect of the loss factors can be evaluated without losing generality by using $\bar{\gamma}_{O i}$ and $\tan \delta_{p}$.

In order to normalize the nondimensional compliance expressed by Eq. (75) with respect to the proposed methods, the following parameters are introduced:

$$
\bar{u}_{L i}=\frac{\frac{\Xi_{i}}{\Xi_{\mathrm{st} i}}}{\sqrt{\frac{2}{\beta_{i}}}}, \quad \bar{G}_{L i}=\frac{g_{i}^{2}-1}{\sqrt{\beta_{i}}}, \bar{\gamma}_{L i}=\frac{\gamma_{i}}{\frac{1}{2} \sqrt{\frac{\beta_{i}}{2}}}, \bar{\delta}_{p i}=\frac{\tan \delta_{p}}{\sqrt{\frac{3 \beta_{i}}{2}}}, \bar{\delta}_{c i}=\frac{\tan \delta_{c}}{\sqrt{\frac{3 \beta_{i}}{2}}} .
$$

Substituting Eqs. (116)-(120) in Eq. (75), we derive the normalized nondimensional compliance as

$$
\bar{u}_{L i} \approx \frac{1}{\sqrt{2}} \frac{1}{-\bar{G}_{L i}+\mathrm{j} \frac{1}{\sqrt{2}} \bar{\gamma}_{L i}+\frac{-1}{-\bar{G}_{L i}+\mathrm{j} \sqrt{\frac{3}{2}}\left(\bar{\delta}_{c i}+\bar{\delta}_{p i}\right)}},
$$

where parameters with negligible values are ignored considering that $\beta_{i}<<\sqrt{\beta_{i}}<<1, \tan \delta_{c}<<1$, and $\tan \delta_{p}<<1$. Because the optimum frequency ratios for both the proposed methods differ by a small amount as indicated by Eq. (93), the normalized nondimensional compliances for both the methods are given by Eq. (121). Equation (121) indicates that the effect of the loss factors can be evaluated using $\bar{\gamma}_{L i}, \bar{\delta}_{p i}$, and $\bar{\delta}_{c i}$. Further, it indicates that the effects of the dielectric loss tangents $\tan \delta_{c}$ and $\tan \delta_{p}$ are almost similar. In practice, a capacitor value with $\tan \delta_{c}$ less than $\tan \delta_{p}$ is usually chosen.

\subsection{Validation of normalization and verification of the effect of loss factors by simulations}

In this subsection, the effect of the modal damping ratio and the loss factors on the measured equivalent stiffness ratios is investigated, and the change in the magnitude plots of the nondimensional compliance is investigated through simulations. In the simulations, a high frequency resolution, which satisfies $A_{r}>10000$, is used to eliminate the errors caused by the frequency resolution. Here, the resolution evaluation index $A_{r}$ is 
calculated using the true value of the equivalent stiffness ratio. The ratio of the measured equivalent stiffness ratio $\hat{\beta}_{i}$ to its true value $\beta_{i}$ is defined as the agreement evaluation index:

$$
A_{a}=\frac{\hat{\beta}_{i}}{\beta_{i}} .
$$

The agreement evaluation indices for the conventional and proposed methods are obtained by simulations using the nondimensional compliances obtained using Eqs. (65) and (75), respectively. Further, the optimum frequency ratios given by Eq. (93) are used in the two proposed methods. In the simulations, $\beta_{i}=0.03, \beta_{i}=0.003$, and $\beta_{i}=0.0003$ are used to validate the normalization.

The simulation results of the agreement evaluation index for the conventional method using different values of $\bar{\gamma}_{o i}$ and $\tan \delta_{p}$ are shown in Fig. 12. The decrease in accuracy is observed to be sufficiently small. The accuracy of the measured equivalent stiffness ratio depends almost exclusively on the dielectric loss tangent $\tan \delta_{p}$. The effect of the modal damping ratio is small when the value of the equivalent stiffness ratio is significantly smaller than 1 . In such cases, normalization is also effective because it causes the difference between the results obtained using $\beta_{i}=0.003$ and $\beta_{i}=0.0003$ to be negligible. The simulated magnitude plots of the nondimensional compliance of the piezoelectric element with short- and open-circuited electrodes are shown in Fig. 13 for $\beta_{i}=0.003$. It is observed that the peaks are obtuse for large values of loss factors. In practice, this obtuseness tends to introduce errors; however, the effect of obtuseness is not taken into consideration in the simulations.

The simulation results of the agreement evaluation index obtained for the two-peak method using different values of $\bar{\gamma}_{L i}$ and $\bar{\delta}_{p i}$ are shown in Fig. 14. However, the simulation results obtained using different values of $\bar{\delta}_{c i}$ are not shown in the figure because they are similar to those obtained using different values of $\bar{\delta}_{p i}$. The normalization is validated because the difference among the simulation results obtained for $\beta_{i}=0.03$, $\beta_{i}=0.003$, and $\beta_{i}=0.0003$ is small. Furthermore, the effects of the modal damping ratio and the dielectric loss tangents are not independent of each other. In the case where the dielectric loss tangents are negligible, the accuracy is not compromised even if the modal damping ratio is large. The simulated magnitude plots of the nondimensional compliance when the inductor is coupled to the piezoelectric element are shown in Fig. 15. Here, only the combination of $\beta_{i}=0.003$ and $\bar{\delta}_{c i}=0$ is used because it represents the general tendency of the loss factors. In addition, the optimum frequency ratio given by Eq. (93) is applied. From the results obtained using the inductive circuit, it is observed that it becomes difficult to distinguish between the two peaks when the dielectric loss tangents are large, and the two peaks combine into one if $\bar{\delta}_{p i}+\bar{\delta}_{c i}$ is slightly greater than 1 . Because the amplitudes of the two peaks are almost equal when the optimum frequency ratio is used, the inductance should be tuned for the experimental measurement such that the two resonance peaks match in amplitude.

The simulation results of the agreement evaluation index obtained for the two-point intersection method using different values of $\bar{\gamma}_{L i}$ and $\bar{\delta}_{p i}$ are shown in Fig. 16. The simulation results obtained using different values of $\bar{\delta}_{c i}$ are not shown in the figure because they are similar to those obtained using different values of 
$\bar{\delta}_{p i}$. These results show the effectiveness of normalization. In the case when the modal damping ratio or the dielectric loss tangents are negligible, the accuracy of the measured equivalent stiffness ratio is maintained. If both of them are not negligible, the measured equivalent stiffness ratio becomes greater than the true value. The simulated magnitude plots of the nondimensional compliance are shown in Fig. 17. Here, $\beta_{i}=0.003$ and the optimum frequency ratio given by Eq. (93) were used. The accuracy of the measured value is not compromised even if $\bar{\delta}_{p i}$ is greater than 1 . This result is obtained under the condition that the modal damping ratio is negligible in theory; however, this method has a disadvantage that the intersection points obtained under the abovementioned condition are unclear. Similar to the two-peak method, even for this method, the inductance should be tuned to achieve two peaks of equal amplitude for the experimental measurements. A comparison between the two proposed methods suggests that the two-point intersection method is better than the two-peak method only when the modal damping ratio is small enough to be neglected.

\subsection{Improved method using negative resistor}

The accuracy of the measured equivalent stiffness ratio for the two proposed methods is compromised owing to the existence of the modal damping ratio and the dielectric loss tangents. The most important cause of this effect is the change in the resonance frequencies and, thus, the intersection points. The second cause is the obtuseness of the peaks and the intersection points in the frequency response. The obtuseness tends to introduce an error in the actual experimental measurements. The measured equivalent stiffness ratio can roughly be corrected by using the results shown in Figs. 14 or 16; however, the accuracy obtained after error correction is moderate because the error due to the obtuseness of the two peaks or the two intersection points cannot be prevented. In order to solve these problems, we propose an improved method that uses a negative resistor. The analytical models of the proposed improved method are shown in Fig. 18. A negative resistor is added to the inductor in parallel or series. The resistances of the piezoelectric element and the simulated inductor are represented separately in this model. The negative resistors must be tuned such that the dielectric loss tangents are canceled. Both the parallel and series configurations can be applied; however, the optimum values of the negative resistances are different for the two configurations. The model of the parallel configuration is identical to that shown in Fig. 11. The nondimensional compliance for the parallel configuration is expressed as

$$
\begin{gathered}
\frac{\Xi_{i}}{\Xi_{\mathrm{st} i}}=\frac{1}{-g_{i}^{2}+2 \mathrm{j} \gamma_{i} g_{i}+1+\beta_{i} \frac{-g_{i}^{2}+\mathrm{j} \tan \delta_{c} \cdot g_{i}^{2}}{\left(-1+\tan \delta_{c} \tan \delta_{p}\right) g_{i}^{2}+\mathrm{j}\left(\tan \delta_{c}+\tan \delta_{p}\right) g_{i}^{2}+2 \mathrm{j} \zeta_{\mathrm{np}} f_{i} g_{i}+f_{i}^{2}+2 \tan \delta_{c} \cdot \zeta_{\mathrm{np}} f_{i} g_{i}}}, \\
\zeta_{\mathrm{np}}=\frac{1}{2 R_{\mathrm{np}}} \sqrt{\frac{L_{g}}{C_{p}^{\prime S}}},
\end{gathered}
$$

where $R_{\mathrm{np}}$ is the resistance of the added negative resistor. Further, the nondimensional compliance for the series configuration is expressed as 


$$
\begin{gathered}
\frac{\Xi_{i}}{\Xi_{\mathrm{st} i}}=\frac{1}{-g_{i}^{2}+2 \mathrm{j} \gamma_{i} g_{i}+1+\beta_{i} \frac{-g_{i}^{2}+\mathrm{j} \tan \delta_{c} \cdot g_{i}^{2}+2 \mathrm{j} \zeta_{\mathrm{ns}} f_{i} g_{i}}{\left(-1+\tan \delta_{c} \tan \delta_{p}\right) g_{i}^{2}+\mathrm{j}\left(\tan \delta_{c}+\tan \delta_{p}\right) g_{i}^{2}+2 \mathrm{j} \zeta_{\mathrm{ns}} f_{i} g_{i}+f_{i}^{2}+2 \tan \delta_{p} \cdot \zeta_{\mathrm{ns}} f_{i} g_{i}}}, \\
\zeta_{\mathrm{ns}}=\frac{R_{\mathrm{ns}}}{2} \sqrt{\frac{C_{p}^{\prime S}}{L_{g}}} .
\end{gathered}
$$

In order to clarify the function for the negative resistor, the normalized nondimensional compliance is expressed as

$$
\begin{aligned}
& \bar{u}_{L i} \approx\left\{\begin{array}{ll}
\frac{1}{\sqrt{2}} \frac{1}{-\bar{G}_{L i}+\mathrm{j} \frac{1}{\sqrt{2}} \bar{\gamma}_{L i}+\frac{1}{-\bar{G}_{L i}+\mathrm{j} \sqrt{\frac{3}{2}}\left(\bar{\delta}_{c i}+\bar{\delta}_{p i}+\bar{\zeta}_{\mathrm{np} i}\right)}} \quad \text { (Parallel) } \\
\frac{1}{\sqrt{2}} \frac{1}{-\bar{G}_{L i}+\mathrm{j} \frac{1}{\sqrt{2}} \bar{\gamma}_{L i}+\frac{-1}{-\bar{G}_{L i}+\mathrm{j} \sqrt{\frac{3}{2}}\left(\bar{\delta}_{c i}+\bar{\delta}_{p i}+\bar{\zeta}_{\mathrm{nsi}}\right)}} \text { (Series) }
\end{array},\right. \\
& \bar{\zeta}_{\mathrm{np} i}=\frac{\zeta_{\mathrm{np}}}{\frac{1}{2} \sqrt{\frac{3 \beta_{i}}{2}}}, \bar{\zeta}_{\mathrm{ns} i}=\frac{\zeta_{\mathrm{ns}}}{\frac{1}{2} \sqrt{\frac{3 \beta_{i}}{2}}},
\end{aligned}
$$

where $\bar{\zeta}_{\mathrm{np} i}$ and $\bar{\zeta}_{\mathrm{ns} i}$ are the normalized resistance ratios for parallel and series configurations, respectively. From Eq. (127), it can be observed that the effect of the dielectric loss tangents can be eliminated using negative resistance. The optimum tuning condition is defined as

$$
\left.\begin{array}{l}
\bar{\zeta}_{\mathrm{npi} i} \\
\bar{\zeta}_{\mathrm{ns} i} \mid
\end{array}\right\}=-\bar{\delta}_{c i}-\bar{\delta}_{p i} .
$$

Under this condition, the system becomes unstable, and the values of $\bar{\zeta}_{\mathrm{np} i}$ and $\bar{\zeta}_{\mathrm{ns} i}$ are slightly greater than $-\bar{\delta}_{c i}-\bar{\delta}_{p i}$.

The simulation results of the agreement evaluation index obtained for the two-peak method using a negative resistor are shown in Fig. 19. For cases where the value of the modal equivalent stiffness ratio is small, only $\beta_{i}=0.0003$ is used in the simulations. This is because the accuracy is largely affected by the dielectric loss tangents. In addition, different values of $\bar{\gamma}_{L i}$ and $\bar{\delta}_{p i}$ were used, and $\bar{\zeta}_{\mathrm{np} i}$ and $\bar{\zeta}_{\mathrm{ns} i}$ were tuned to $-\bar{\delta}_{p i}+0.02$. However, the simulation results using different values of $\bar{\delta}_{c i}$ are not shown in the figure because they are similar to those obtained using different values of $\bar{\delta}_{p i}$. The accuracy of the equivalent stiffness ratio obtained using the two-peak method is significantly improved even if $\bar{\delta}_{p i}$ is greater than 1 . The simulated magnitude plots of the nondimensional compliance are shown in Fig. 20. Here, $\beta_{i}=0.0003$ and the optimum frequency ratio given by Eq. (93) were used. The two peaks can be clearly observed by using the negative resistors. This improvement can also be applied to the two-point intersection method; however, the effectiveness of this method is not verified here because the accuracy of the equivalent stiffness ratio obtained using the two-point intersection method is, in general, lower than that obtained for the two-peak method when the 
dielectric loss tangents are sufficiently small.

\section{Validation of proposed methods through experiment}

\subsection{Experimental apparatus}

A schematic of the experimental apparatus is shown in Fig. 21. The material properties of the experimental apparatus are listed in Table 1. Here $m_{\text {acc }}$ is the mass of the accelerometer, $x_{\text {acc }}$ is the $x$ coordinate of the accelerometer, $E_{a}$ is the Young's modulus of the adhesive bond, and $v_{a}$ is the Poisson's ratio of the adhesive bond. The estimated average strain ratio $b=0$ is given in Table 1 because the width of the cantilever was much larger than that of the piezoelectric element. Two piezoelectric elements were attached to the cantilever. The modal equivalent stiffness ratios of each piezoelectric element were measured by exciting the cantilever using the other piezoelectric element. For convenience, the two elements are referred to as A and B. The experimental results obtained using piezoelectric element $B$ are not provided except for necessary information because there is no essential difference between piezoelectric elements A and B. The piezoelectric elements were attached to the cantilever using conductive and nonconductive adhesive bonds. A drop of conductive adhesive was placed on the center of the piezoelectric elements, and this drop was surrounded by a nonconductive adhesive. The conductive adhesive was used to simplify wiring, and the nonconductive one was used for electrical insulation. Cyanoacrylate was used as the nonconductive adhesive because it requires a short curing time and has low viscosity. These properties of cyanoacrylate are advantageous for the preparation of a thin bonding layer. For simplicity, the effect of the conductive adhesive bond was considered to be the same as that considered for the cyanoacrylate adhesive bond in the theoretical estimation. In addition, the bonding layer was assumed to be uniform. The value of the Poisson's ratio of the acrylate resin instead of that of the actual adhesive bond is given in Table 1. The asterisks * against some of the values listed in Table 1 denote that these values are derived using the Poisson's ratio of the acrylate resin. The modal equivalent stiffness ratios for the first five vibration modes were measured in this experiment. A schematic of the negative resistors used in the experiment is shown in Fig. 22. A negative impedance converter was used to obtain negative resistance. The resistance of the negative resistors in parallel and series configurations is given as

$$
\left.\begin{array}{c}
R_{\mathrm{np}} \\
R_{\mathrm{ns}}
\end{array}\right\}=-\frac{R_{n 1} R_{n 3}}{R_{n 2}} .
$$

The arrangement of the input stage of the operational amplifier is determined by the stability condition. The value of the negative resistance was adjusted using a variable resistor. The generalized impedance converter shown in Fig. 10 was used for the simulated inductor in the experiments, wherein $R_{5}$ was variable. 
Table 1

Material properties of cantilever, accelerometer, piezoelectric elements, and bonding layer. $*$ denotes the values derived using the Poisson's ratio of acrylate resin.

\begin{tabular}{|c|c|c|c|c|}
\hline \multirow{6}{*}{ Cantilever } & Length & $l_{b}$ & 0.280 & $\mathrm{~m}$ \\
\hline & Width & $w_{b}$ & 0.0499 & $\mathrm{~m}$ \\
\hline & Thickness & $t_{b}$ & 0.00293 & $\mathrm{~m}$ \\
\hline & Density & $\rho_{b}$ & 7900 & $\mathrm{~kg} / \mathrm{m}^{3}$ \\
\hline & Young's modulus & $E_{b}$ & $2.06 \times 10^{11}$ & $\mathrm{~N} / \mathrm{m}^{2}$ \\
\hline & Average strain ratio & $b$ & 0 & \\
\hline \multirow{2}{*}{ Accelerometer } & Mass & $m_{\mathrm{acc}}$ & 0.0020 & $\mathrm{~kg}$ \\
\hline & Location & $x_{\text {acc }}$ & 0.270 & $\mathrm{~m}$ \\
\hline \multirow{10}{*}{ Piezoelectric element } & Length & $l_{p}$ & 0.0320 & $\mathrm{~m}$ \\
\hline & Width & $w_{p}$ & 0.0220 & $\mathrm{~m}$ \\
\hline & Thickness & $t_{p}$ & 0.00023 & $\mathrm{~m}$ \\
\hline & Density & $\rho_{p}$ & 8050 & $\mathrm{~kg} / \mathrm{m}^{3}$ \\
\hline & Young's modulus & $E_{p}$ & $6.45 \times 10^{10}$ & $\mathrm{~N} / \mathrm{m}^{2}$ \\
\hline & Piezoelectric constant & $d_{31}$ & $2.30 \times 10^{-10}$ & $\mathrm{C} / \mathrm{N}$ \\
\hline & Electrical permittivity & $\varepsilon_{33}^{T}$ & $2.35 \times 10^{-8}$ & $\mathrm{~F} / \mathrm{m}$ \\
\hline & Poisson's ratio & $v_{p}$ & 0.30 & \\
\hline & Location of piezoelectric element A & $\left(x_{L}, x_{R}\right)$ & $(0.0050,0.0370)$ & $\mathrm{m}$ \\
\hline & Location of piezoelectric element B & $\left(x_{L}, x_{R}\right)$ & $(0.0400,0.0720)$ & $\mathrm{m}$ \\
\hline \multirow{5}{*}{ Bonding layer } & Thickness & $t_{a}$ & 0.00010 & $\mathrm{~m}$ \\
\hline & Young's modulus & $E_{a}$ & $9.80 \times 10^{8}$ & $\mathrm{~N} / \mathrm{m}^{2}$ \\
\hline & Poisson's ratio (acrylate resin) & $v_{a}$ & 0.35 & \\
\hline & Transverse elasticity modulus & $G_{a}$ & $3.6 \times 10^{8} *$ & $\mathrm{~N} / \mathrm{m}^{2}$ \\
\hline & Shear lag parameter & $\lambda$ & $500 *$ & $\mathrm{~m}^{-1}$ \\
\hline
\end{tabular}

\subsection{Theoretical estimation}

The spring constant ratios $r_{a 1}$ and $r_{a 2}$, which are derived using Eq. (2) and the material properties of the experimental apparatus, are listed in Table 2. Further, the values of $E_{p}^{\prime}, d_{31}^{\prime}$, and $\varepsilon_{33}^{\prime T}$ obtained using Eqs. (27)-(29) are also listed in Table 2. Because accurate mode shapes are essential for theoretically obtaining accurate modal equivalent stiffness ratios, a calculation model for the cantilever is constructed, as shown in Fig. 23. The cantilever is divided into 280 segments, and the equivalent spring constant and mass of each segment were derived. The stiffness and the mass of the two piezoelectric elements along with the mass of the accelerometer were considered. 
The theoretically calculated modal equivalent stiffness ratios $\beta_{i}$, modal electromechanical coupling coefficients $\Theta_{i}$, capacitance $C_{p}^{\prime S}$, modal stiffness $K_{i}$, the apparent elemental electromechanical coupling coefficient in the longitudinal direction $\theta_{p 1}^{\prime}$, and the modal influence coefficient of the piezoelectric element $\theta_{k i}$ are listed in Table 3. Here, $\psi_{i}$ is positive in the downward direction, and the mode shape functions whose $\psi_{i}\left(l_{b}\right)$ are positive are used. In addition, symbols (s) and (c) denote simplex and complex deformations, respectively. The values of $\psi_{i}\left(x_{\text {acc }}\right)$ are also listed in Table 3 because these values are required to derive the modal displacement in the subsequent experiments.

Table 2

Spring constant ratios and apparent material properties of piezoelectric elements used in the experiment.

\begin{tabular}{cccl}
\hline \multirow{2}{*}{ Spring constant ratio } & $r_{a 1}$ & \multicolumn{2}{c}{6.8} \\
\cline { 2 - 4 } & $r_{a 2}$ & \multicolumn{2}{c}{4.4} \\
\hline Apparent Young's modulus & $E_{p}^{\prime}$ & $6.0 \times 10^{10}$ & $\mathrm{~N} / \mathrm{m}^{2}$ \\
\hline Apparent piezoelectric constant & $d_{31}^{\prime}$ & $2.9 \times 10^{-10}$ & $\mathrm{C} / \mathrm{N}$ \\
\hline Apparent electrical permittivity & $\varepsilon_{33}^{\prime T}$ & $2.1 \times 10^{-8}$ & $\mathrm{~F} / \mathrm{m}$ \\
\hline
\end{tabular}

Table 3

Theoretically calculated properties of piezoelectric element A.

\begin{tabular}{|c|c|c|c|c|c|c|c|c|}
\hline Mode & $(\mathrm{s})$ or $(\mathrm{c})$ & $\beta_{i}$ & $\begin{array}{c}\Theta_{i} \\
(\mathrm{~N} / \mathrm{V}) \\
\end{array}$ & $\begin{array}{l}C_{p}^{\prime S} \\
(\mathrm{~F})\end{array}$ & $\begin{array}{c}K_{i} \\
(\mathrm{~N} / \mathrm{m})\end{array}$ & $\begin{array}{c}\theta_{p 1}^{\prime} \\
(\mathrm{N} / \mathrm{V}) \\
\end{array}$ & $\theta_{k i}$ & $\psi_{i}\left(x_{\mathrm{acc}}\right)$ \\
\hline 1 & (s) & 0.0038 & 0.0026 & \multirow{5}{*}{$4.8 \times 10^{-8}$} & 37500 & \multirow{5}{*}{0.38} & 0.00691 & 3.31 \\
\hline 2 & (s) & 0.0021 & -0.012 & & 1460000 & & -0.0321 & 2.83 \\
\hline 3 & (s) & 0.0011 & 0.025 & & 11400000 & & 0.0662 & 2.42 \\
\hline 4 & (c) & 0.00028 & -0.024 & & 43900000 & & -0.0642 & 2.02 \\
\hline 5 & (c) & 0.0000031 & 0.0043 & & 120000000 & & 0.0113 & 1.64 \\
\hline
\end{tabular}

\subsection{Measurement in experiments}

The experimental results of the nondimensional compliance when the electrodes of the piezoelectric element A were short- and open-circuited are shown in Fig. 24. In order to derive the nondimensional compliance, the modal displacement and the static modal displacement are required. In this study, the modal displacement is expressed as

$$
\xi_{i}=\frac{W\left(x_{\mathrm{acc}}, t\right)}{\psi_{i}\left(x_{\mathrm{acc}}\right)},
$$

where $W\left(x_{\text {acc }}, t\right)$ is the displacement of the cantilever with respect to the position of the accelerometer. $W\left(x_{\text {acc }}, t\right)$ was obtained from accelerometer measurements, and the values of $\psi_{i}\left(x_{\text {acc }}\right)$ that are listed in Table 3 
were obtained by theoretical calculation. Because the excitation force was applied using the other piezoelectric element, the modal electromechanical coupling coefficient is required to derive the static modal displacement. The values of the modal electromechanical coupling coefficients, which are derived later in this section and are listed in Table 15, were used. However, it should be noted that the modal equivalent stiffness ratios can be obtained without an accurate electromechanical coupling coefficient of the piezoelectric element used for excitation. Furthermore, the modal mass $M_{i}$, modal damping coefficient $D_{i}$, modal stiffness $K_{i}$, modal damping ratio $\gamma_{i}$, natural frequency $F_{S i}$ with short-circuited electrodes, and frequency resolution $\Delta F$ obtained in the experiment are listed in Table 4 . The natural frequencies listed in Table 4 are the damped natural frequencies. The modal stiffness was approximately derived as

$$
K_{i}=\left(2 \pi F_{S i}\right)^{2} .
$$

The dielectric loss tangent $\tan \delta_{p}$ and capacitance $C_{p}^{\prime S}$ were measured for the natural frequencies of the vibration modes, and their values are listed in Table 5. When $\tan \delta_{p}$ and $C_{p}^{\prime S}$ of the attached piezoelectric elements were measured, the host structure was not clamped so that the effect of the resonance of the host structure was eliminated. Capacitance $C_{p}^{\prime S}$ decreases by a small amount as the frequency increases. This effect is attributed to the viscoelastic property of the adhesive bond used in the experiment. Moreover, it can be observed from Eq. (1) that the spring constant of the adhesive bond increases with an increase in frequency although viscosity was not taken into consideration in this equation. The experimental results for the nondimensional compliance when the inductor was coupled to the piezoelectric element are shown in Fig. 25. These results were used in the two-peak method as well as the two-point intersection method. The dielectric loss $\operatorname{tangent} \tan \delta_{c}$ measured at the natural frequencies of the vibration modes are also listed in Table 5. Further, the experimental results obtained by using the negative resistors are shown in Fig. 26. In this figure, the noise generated at approximately $540 \mathrm{~Hz}$ is attributed to the ninth-order power line harmonics. The values of the inductance, negative resistance, frequency ratio, and resistance ratio are listed in Tables 6-8. It should be noted that the values with respect to the electrical circuits are not necessary for obtaining the modal equivalent stiffness ratios and the modal electromechanical coupling coefficients. However, these values are presented here for reference. The values of resonance frequencies $F_{L i}$ and $F_{R i}$, frequencies of the intersection points $F_{P i}$ and $F_{Q i}$, and clearly visible resonance frequencies $F_{L i}$ and $F_{R i}$ obtained using negative resistors are listed in Tables 9 and 10. In addition, the natural frequency $F_{O i}$ when the electrodes of the piezoelectric element were in an open-circuit state is given in Table 9. The natural frequency $F_{S i}$ when the electrodes of the piezoelectric element were in a short-circuit state and the frequency resolution are listed in Tables 9 and 10, for convenience. The modal equivalent stiffness ratios derived by the three methods using the frequencies listed in Tables 9 and 10 are given in Table 11. The resolution evaluation index is listed in Table 12. The significant values of the equivalent stiffness ratios were determined on the basis of their resolution evaluation indices. A comparison of the resolution evaluation index for the conventional method and the proposed ones indicates that both the proposed methods are much more accurate than the conventional one. Further, the improvement resulting from the use of negative resistors is notable. Even if the two peaks cannot be observed clearly because of the effect of 
dielectric loss tangents, the modal equivalent stiffness ratio can be obtained using the negative resistor. In order to evaluate the effect of the modal damping ratio and the dielectric loss tangents, the average values of the modal equivalent stiffness ratios obtained using the negative resistors in parallel and series are used as the preliminary true values. The preliminary true values and the normalized values are listed in Table 13 . The values of dielectric loss tangent $\tan \delta_{p}$ are also given in Table 13, for convenience. The values of the normalized negative resistance ratios are approximately equal to the sum of the normalized dielectric loss tangent ratios; however, the absolute values of the normalized negative resistance ratios are slightly larger than the sum of the normalized dielectric loss tangent ratios in many cases. Even in such cases, the system does not lose stability because the modal damping ratio is sufficiently large. The values of the results listed in Table 13 indicate that the condition under which the normalized damping ratio $\bar{\gamma}_{L i}$ is small and the sum of the normalized dielectric loss tangents, $\bar{\delta}_{p i}$ and $\bar{\delta}_{c i}$, is large is not investigated thus far. Subsequently, an experiment with a resistor added in parallel to the inductor was performed to simulate the large dielectric loss tangents. The experimental results of the nondimensional compliance using the additional resistor are shown in Fig. 27. Here, $R_{\text {ap }}$ denotes the resistance of the additional resistor, and the first vibration mode was used for the simulation. The improved method was not used in this experiment because it obviously works well. The values of the circuit elements, important frequencies, and modal equivalent stiffness ratios for this experiment are listed in Table 14. The results of the two-point intersection method are found to be more accurate than those of the two-peak method. The results demonstrate the value of the two-point intersection method.

The modal electromechanical coupling coefficients obtained using the preliminary true values of the modal equivalent stiffness ratio, capacitance, and modal stiffness in the experiments are given in Table 15. In this table, the signs of the values were determined from the phase plots of the compliance when the piezoelectric elements were used for excitation. These values were used to obtain the nondimensional compliance shown in Figs. 24-27, as already mentioned. The theoretical estimation of the modal equivalent stiffness ratios and the modal electromechanical coupling coefficients agrees well with the experimental results in some cases. This good agreement validates the theoretical analysis with respect to the effect of the adhesive bonds. However, in several cases, there is a large difference in the theoretical and practical results. The disagreements occur particularly in cases wherein the value of the equivalent stiffness ratio is small. This indicates that the disagreements occur owing to a mismatch in the experimentally measured and theoretically calculated mode shapes. In the example illustrated in this study, not only the values but also the signs of the experimentally and theoretically obtained electromechanical coupling coefficients differed for the fifth vibration mode. These results indicate that experimental measurement is essential for accurately obtaining the modal equivalent stiffness ratio and modal electromechanical coupling coefficient even if the host structure is simple and the mode shape function can be derived in a relatively precise manner. 
Table 4

Experimentally measured properties of cantilever when the electrodes of piezoelectric element A were short-circuited.

\begin{tabular}{|c|c|c|c|c|c|c|}
\hline Mode & $M_{i}(\mathrm{~kg})$ & $D_{i} \quad(\mathrm{Ns} / \mathrm{m})$ & $K_{i}(\mathrm{~N} / \mathrm{m})$ & $\gamma_{i}$ & $F_{S i}(\mathrm{~Hz})$ & $\Delta F \quad(\mathrm{~Hz})$ \\
\hline 1 & \multirow{5}{*}{$\begin{array}{c}1 \\
\text { (defined) }\end{array}$} & 0.23 & 37200 & 0.00060 & 30.68 & 0.02 \\
\hline 2 & & 1.3 & 1450000 & 0.00054 & 191.64 & 0.04 \\
\hline 3 & & 6.5 & 11420000 & 0.00096 & 537.80 & 0.05 \\
\hline 4 & & 19 & 43807000 & 0.0014 & 1053.4 & 0.1 \\
\hline 5 & & 20 & 119600000 & 0.00091 & 1740.8 & 0.2 \\
\hline
\end{tabular}

Table 5

Experimentally measured values of dielectric loss tangent and capacitance of piezoelectric element A and dielectric loss tangents of capacitors for different vibration modes.

\begin{tabular}{cccc}
\hline Mode & $\tan \delta_{p}$ & $C_{p}^{\prime S}(\mathrm{~F})$ & $\tan \delta_{c}$ \\
\hline 1 & 0.020 & $4.96 \times 10^{-8}$ & 0.0013 \\
\hline 2 & 0.019 & $4.86 \times 10^{-8}$ & 0.0026 \\
\hline 3 & 0.021 & $4.73 \times 10^{-8}$ & 0.0044 \\
\hline 4 & 0.019 & $4.67 \times 10^{-8}$ & 0.0065 \\
\hline 5 & 0.021 & $4.68 \times 10^{-8}$ & 0.0086 \\
\hline
\end{tabular}

Table 6

Inductance and frequency ratio values obtained with the inductor coupled to the piezoelectric element $\mathrm{A}$ in the experiment.

\begin{tabular}{ccc}
\hline Mode & $L_{g}(\mathrm{H})$ & $f_{i}$ \\
\hline 1 & 552 & 0.991 \\
\hline 2 & 14.3 & 0.996 \\
\hline 3 & 1.91 & 0.985 \\
\hline 4 & 0.502 & 0.987 \\
\hline 5 & 0.183 & 0.988 \\
\hline
\end{tabular}




\section{Table 7}

Values of circuit parameters for the improved two-peak method, with a negative resistor connected in parallel to the inductor for the experiment using piezoelectric element A.

\begin{tabular}{ccccc}
\hline Mode & $L_{g}(\mathrm{H})$ & $R_{\mathrm{np}}(\Omega)$ & $f_{i}$ & $\zeta_{\mathrm{np}}$ \\
\hline 1 & 555 & -4620000 & 0.989 & -0.0114 \\
\hline 2 & 13.9 & -858000 & 1.01 & -0.00986 \\
\hline 3 & 1.94 & -253300 & 0.977 & -0.0126 \\
\hline 4 & 0.502 & -104100 & 0.987 & -0.0157 \\
\hline 5 & 0.183 & -64600 & 0.988 & -0.0153 \\
\hline
\end{tabular}

Table 8

Values of circuit parameters for the improved two-peak method, with a negative resistor connected in series to the inductor for the experiment using piezoelectric element $\mathrm{A}$.

\begin{tabular}{ccccc}
\hline Mode & $L_{g}(\mathrm{H})$ & $R_{\mathrm{ns}}(\Omega)$ & $f_{i}$ & $\zeta_{\mathrm{ns}}$ \\
\hline 1 & 563 & -3170 & 0.982 & -0.0149 \\
\hline 2 & 14.1 & -192.9 & 1.00 & -0.00566 \\
\hline 3 & 1.94 & -140.1 & 0.977 & -0.0109 \\
\hline 4 & 0.504 & -83.4 & 0.985 & -0.0127 \\
\hline 5 & 0.184 & -56.7 & 0.985 & -0.0143 \\
\hline
\end{tabular}

Table 9

Resonance frequencies used in conventional, two-peak, and two-point intersection methods for the experiment using piezoelectric element $\mathrm{A}$.

\begin{tabular}{cccccccc}
\hline Mode & $\begin{array}{c}\Delta F \\
(\mathrm{~Hz})\end{array}$ & $\begin{array}{c}F_{S i} \\
(\mathrm{~Hz})\end{array}$ & $\begin{array}{c}F_{O i} \\
(\mathrm{~Hz})\end{array}$ & $\begin{array}{c}F_{L i} \\
(\mathrm{~Hz})\end{array}$ & $\begin{array}{c}F_{R i} \\
(\mathrm{~Hz})\end{array}$ & $\begin{array}{c}F_{P i} \\
(\mathrm{~Hz})\end{array}$ & $\begin{array}{c}F_{Q i} \\
(\mathrm{~Hz})\end{array}$ \\
\hline 1 & 0.02 & 30.68 & 30.74 & 29.80 & 31.70 & 30.04 & 31.38 \\
\hline 2 & 0.04 & 191.64 & 191.80 & 188.56 & 196.76 & 189.12 & 194.60 \\
\hline 3 & 0.05 & 537.80 & 537.90 & 532.10 & 543.75 & 532.75 & 543.30 \\
\hline 4 & 0.1 & 1053.4 & 1053.5 & - & - & 1048.6 & 1059.7 \\
\hline 5 & 0.2 & 1740.8 & 1740.8 & - & - & 1731.4 & 1749.6 \\
\hline
\end{tabular}


Table 10

Resonance frequencies used in the two-peak method with a negative resistor for the experiment using piezoelectric element A.

\begin{tabular}{ccccccc}
\hline \multirow{2}{*}{ Mode } & $\Delta F(\mathrm{~Hz})$ & $F_{S i}(\mathrm{~Hz})$ & \multicolumn{2}{c}{$\begin{array}{c}\text { Negative resistor } \\
\text { connected in parallel }\end{array}$} & \multicolumn{2}{c}{$\begin{array}{c}\text { Negative resistor } \\
\text { connected in series }\end{array}$} \\
\cline { 4 - 7 } & & & $F_{L i}(\mathrm{~Hz})$ & $F_{R i}(\mathrm{~Hz})$ & $F_{L i}(\mathrm{~Hz})$ & $F_{R i}(\mathrm{~Hz})$ \\
\hline 1 & 0.02 & 30.68 & 29.72 & 31.60 & 29.52 & 31.46 \\
\hline 2 & 0.04 & 191.64 & 189.32 & 198.76 & 189.00 & 197.88 \\
\hline 3 & 0.05 & 537.80 & 528.10 & 542.75 & 527.95 & 542.75 \\
\hline 4 & 0.1 & 1053.4 & 1048.8 & 1057.5 & 1049.2 & 1057.7 \\
\hline 5 & 0.2 & 1740.8 & 1736.4 & 1749.6 & 1736.2 & 1749.2 \\
\hline
\end{tabular}

\section{Table 11}

Modal equivalent stiffness ratios of piezoelectric element A derived experimentally using the three methods.

\begin{tabular}{cccccc}
\hline \multirow{2}{*}{ Mode } & \multirow{2}{*}{ Conventional method } & \multicolumn{3}{c}{ Two-peak method } & \multirow{2}{*}{ Two-point intersection method } \\
\cline { 3 - 5 } & & Normal & with $R_{\mathrm{np}}$ & with $R_{\mathrm{ns}}$ & \\
\hline 1 & 0.004 & 0.0038 & 0.0037 & 0.0038 & 0.0038 \\
\hline 2 & 0.002 & 0.0017 & 0.0018 & 0.0018 & 0.0016 \\
\hline 3 & 0.0004 & 0.00047 & 0.00066 & 0.00067 & 0.00077 \\
\hline 4 & 0.0002 & - & 0.000068 & 0.000065 & 0.00022 \\
\hline 5 & 0 & - & 0.000051 & 0.000051 & 0.00022 \\
\hline
\end{tabular}

\section{Table 12}

Resolution evaluation indices of equivalent stiffness ratios listed in Table 11.

\begin{tabular}{cccccc}
\hline \multirow{2}{*}{ Mode } & \multirow{2}{*}{ Conventional method } & \multicolumn{3}{c}{ Two-peak method } & \multirow{2}{*}{ Two-point intersection method } \\
\cline { 2 - 5 } & & Normal & with $R_{\mathrm{np}}$ & with $R_{\mathrm{ns}}$ & \\
\hline 1 & 3 & 47.4 & 47.0 & 47.6 & 33.5 \\
\hline 2 & 4 & 99.3 & 102 & 101 & 68.3 \\
\hline 3 & 2 & 116 & 139 & 140 & 105 \\
\hline 4 & 1 & - & 43.4 & 42.5 & 55.0 \\
\hline 5 & 0 & - & 31.1 & 31.1 & 45.5 \\
\hline
\end{tabular}


Table 13

Normalized values obtained by using preliminary true values of equivalent stiffness ratios in the experiment using piezoelectric element $\mathrm{A}$.

\begin{tabular}{ccccccccc}
\hline \multirow{2}{*}{ Mode } & Preliminary true & \multicolumn{6}{c}{ Conventional method } & \multicolumn{5}{c}{ Proposed methods } \\
\cline { 2 - 9 } & value of $\beta_{i}$ & $\bar{\gamma}_{o i}$ & $\tan \delta_{p}$ & $\bar{\gamma}_{L i}$ & $\bar{\delta}_{p i}$ & $\bar{\delta}_{c i}$ & $\bar{\zeta}_{\mathrm{np} i}$ & $\bar{\zeta}_{\mathrm{ns} i}$ \\
\hline 1 & 0.0038 & 0.63 & 0.020 & 0.028 & 0.26 & 0.017 & -0.30 & -0.39 \\
\hline 2 & 0.0018 & 1.2 & 0.019 & 0.036 & 0.37 & 0.050 & -0.38 & -0.22 \\
\hline 3 & 0.00067 & 5.7 & 0.021 & 0.10 & 0.66 & 0.14 & -0.79 & -0.69 \\
\hline 4 & 0.000067 & 84 & 0.019 & 0.48 & 1.9 & 0.65 & -3.1 & -2.5 \\
\hline 5 & 0.000051 & 71 & 0.021 & 0.36 & 2.4 & 0.98 & -3.5 & -3.3 \\
\hline
\end{tabular}

Table 14

Values of circuit elements, important frequencies, and modal equivalent stiffness ratios for the method with additional resistor connected in parallel to the inductor in the experiment using piezoelectric element A.

\begin{tabular}{|c|c|c|c|c|c|c|c|}
\hline \multirow{2}{*}{$L_{g} \quad(\mathrm{H})$} & \multirow{2}{*}{$R_{\text {ap }}(\Omega)$} & \multicolumn{3}{|c|}{ Two-peak method } & \multicolumn{3}{|c|}{ Two-point intersection method } \\
\hline & & $F_{L 1}(\mathrm{~Hz})$ & $F_{R 1}(\mathrm{~Hz})$ & $\beta_{1}$ & $F_{P 1}(\mathrm{~Hz})$ & $F_{Q 1}(\mathrm{~Hz})$ & $\beta_{1}$ \\
\hline 552 & $3.0 \times 10^{6}$ & 29.88 & 31.62 & 0.0032 & 30.04 & 31.40 & 0.0039 \\
\hline 552 & $1.0 \times 10^{6}$ & - & - & - & 30.04 & 31.40 & 0.0039 \\
\hline
\end{tabular}

Table 15

Modal electromechanical coupling coefficients derived by using preliminary true values of modal equivalent stiffness ratios, capacitance, and modal stiffness for both the piezoelectric elements.

\begin{tabular}{ccc}
\hline \multirow{2}{*}{ Mode } & \multicolumn{2}{c}{$\Theta_{i}(\mathrm{~N} / \mathrm{V})$} \\
& Piezoelectric element A & Piezoelectric element B \\
\hline 1 & 0.0026 & 0.0020 \\
\hline 2 & -0.011 & -0.0007 \\
\hline 3 & 0.019 & -0.021 \\
\hline 4 & -0.012 & 0.0605 \\
\hline 5 & -0.017 & -0.0806 \\
\hline
\end{tabular}

\section{Conclusion}

The governing equations for passive vibration suppression and active vibration control were theoretically formulated by considering the effect of the restraint imposed by the adhesive bonds on the piezoelectric element. Two methods for measuring the equivalent stiffness ratios have been proposed, and their tolerance to measurement error and frequency resolution was theoretically investigated by comparing them with the 
conventional method. Because the accuracy of the equivalent stiffness ratios obtained using the proposed methods is compromised when the loss factors are not sufficiently small, an improved method using a negative resistor has been proposed. These methods were validated through experiments. Further, the accuracy of the theoretical estimations was improved by taking into consideration the restraint imposed by the adhesive bonds. The necessity of experimental measurements for further accuracy improvement was also demonstrated. Moreover, the experimental results are significant because they do not require mode shape functions and circuit element values. Further, it has been shown that these methods provide accurate modal equivalent stiffness ratios and electromechanical coupling coefficients particularly when conventional methods yield poor results.

\section{Acknowledgement}

This study was partly supported by Grant-in-Aid for Young Scientists (B) (JSPS, No. 21760169).

\section{References}

[1] K. Yamada, H. Matsuhisa, H. Utsuno and K. Sawada, Optimum tuning of series and parallel LR circuits for passive vibration suppression using piezoelectric elements, Journal of Sound and Vibration 329 (24) (2010) 5036-5057.

[2] N. W. Hagood and A. Von Flotow, Damping of structural vibrations with piezoelectric materials and passive electrical networks, Journal of Sound and Vibration 146 (2) (1991) 243-268.

[3] C. H. Park, Dynamics modeling of beams with shunted piezoelectric elements, Journal of Sound and Vibration 268 (1) (2003) 115-129.

[4] S. Y. Wu, Piezoelectric shunts with a parallel R-L circuit for structural damping and vibration control, Proceedings of the SPIE 2720, San Diego, February 1996, 259-269.

[5] C. H. Park and D. J. Inman, A uniform model for series R-L and parallel R-L shunt circuits and power consumption, Proceedings of the SPIE 3668, California, March 1999, 797-804.

[6] R. L. Forward, Electronic damping of vibrations in optical structures, Applied Optics 18 (5) (1979) 690-697.

[7] J. J. Dosch, D. J. Inman and E. Garcia, A self-sensing piezoelectric actuator for collocated control, Journal of Intelligent Material Systems and Structures 3 (1992) 166-185.

[8] G. S. Agnes, Development of a modal model for simultaneous active and passive piezoelectric vibration suppression, Journal of Intelligent Material Systems and Structures 6 (1995) 482-487.

[9] M. S. Tsai and K. W. Wang, On the structural damping characteristics of active piezoelectric actuators with passive shunt, Journal of Sound and Vibration 221 (1) (1999) 1-22.

[10] K. Adachi, Y. Awakura and T. Iwatsubo, Hybrid piezoelectric damping for structural vibration suppression, Journal of Intelligent Material Systems and Structures 15 (2004) 795-801. 
[11] K. Yamada, H. Matsuhisa, H. Utsuno and J. G. Park, Hybrid vibration suppression of flexible structures using piezoelectric elements and analog circuits, JSME Journal of Environment and Engineering 3 (2) (2008) 424-435.

[12] K. Yamada, H. Matsuhisa, H. Utsuno and J. G. Park, Precise measurement technique of the electromechanical coupling coefficient of piezoelectric elements, Proceedings of the 8th International Conference on Motion and Vibration Control, Daejeon, August 2006, Flash memory MA1-3.

[13] M. Porfiri, C. Maurini and J. Pouget, Identification of electromechanical modal parameters of linear piezoelectric structures, Smart Materials and Structures 16 (2007) 323-331.

[14] S. Bhalla and C. K. SOH, Electromechanical impedance modeling for adhesively bonded piezo-transducers, Journal of Intelligent Material Systems and Structures 15 (2004) 955-972.

[15] J. Sirohi and I. Chopra, Fundamental understanding of piezoelectric strain sensors, Journal of Intelligent Material and Structures 11 (2000) 246-257.

[16] A. R. de Faria, The impact of finite-stiffness bonding on the sensing effectiveness of piezoelectric patches, Smart Materials and Structures 12 (2003) N5-N8.

\section{List of figure captions}

Fig. 1. Schematic diagrams of plate-type piezoelectric element: (a) plate-type piezoelectric element and (b) piezoelectric element attached to host structure by adhesive bond.

Fig. 2. Analytical model of the stiffness of the bonding layer using equivalent springs: (a) one-dimensional model and (b) two-dimensional model.

Fig. 3. Equivalent mechanical model of piezoelectric element and its lever: (a) $\bar{\theta}_{p 1}^{\prime} \geq 0$ and (b) $\bar{\theta}_{p 1}^{\prime}<0$.

Fig. 4. Equivalent mechanical model without levers.

Fig. 5. Analytical model of passive vibration suppression system using piezoelectric element and electrical impedance.

Fig. 6. Conceptual diagram of complex deformation of piezoelectric element.

Fig. 7. Equivalent mechanical models for targeted vibration mode when the piezoelectric element is shunted with electrical impedance: (a) equivalent model with levers of $\theta_{k i}^{j}>0$ and $\theta_{p 1}^{\prime}>0$ and (b) equivalent model without virtual levers.

Fig. 8. Equivalent circuit of piezoelectric element with dielectric loss.

Fig. 9. Analytical model of two-peak method. 
Fig. 10. Schematic diagram of the simulated inductor using generalized impedance converter and its equivalent circuit: (a) simulated inductor circuit and (b) equivalent circuit.

Fig. 11. Analytical model of two-point intersection method.

Fig. 12. Simulation results of the agreement evaluation index for conventional method using different values of $\bar{\gamma}_{o i}$ and $\tan \delta_{p}:$ (a) $\beta_{i}=0.03$, (b) $\beta_{i}=0.003$, and (c) $\beta_{i}=0.0003$.

Fig. 13. Simulated magnitude plots of nondimensional compliance using $\beta_{i}=0.003$ : (a) short-circuited electrodes with varying $\bar{\gamma}_{O i}$, (b) open-circuited electrodes with $\tan \delta_{p}=0$ and varying $\bar{\gamma}_{O i}$, (c) open-circuited electrodes with $\bar{\gamma}_{O i}=0$ and varying $\tan \delta_{p}$, and (d) open-circuited electrodes with $\bar{\gamma}_{O i}=0.2$ and $\tan \delta_{p}=0.1$.

Fig. 14. Simulation results of the agreement evaluation index for two-peak method using different values of $\bar{\gamma}_{L i}$ and $\bar{\delta}_{p i}$ : (a) $\beta_{i}=0.03$, (b) $\beta_{i}=0.003$, and (c) $\beta_{i}=0.0003$.

Fig. 15. Simulated magnitude plots of nondimensional compliance using $\beta_{i}=0.003$ : (a) short-circuited electrodes with varying $\bar{\gamma}_{L i}$, (b) inductive circuit with $\bar{\delta}_{p i}=0$ and varying $\bar{\gamma}_{L i}$, (c) inductive circuit with $\bar{\gamma}_{L i}=0$ and varying $\bar{\delta}_{p i}$, and (d) inductive circuit with various combinations of $\bar{\gamma}_{L i}$ and $\bar{\delta}_{p i}$.

Fig. 16. Simulation results of agreement evaluation index for two-point intersection method using different values of $\bar{\gamma}_{L i}$ and $\bar{\delta}_{p i}:$ (a) $\beta_{i}=0.03$, (b) $\beta_{i}=0.003$, and (c) $\beta_{i}=0.0003$.

Fig. 17. Simulated magnitude plots of nondimensional compliance for $\beta_{i}=0.003$ : (a) $\bar{\delta}_{p i}=0$ and varying $\bar{\gamma}_{L i}$, (b) $\bar{\gamma}_{L i}=0$ and varying $\bar{\delta}_{p i}$, (c) $\bar{\gamma}_{L i}=\bar{\delta}_{p i}=0.3$, and (d) $\bar{\gamma}_{L i}=\bar{\delta}_{p i}=1$.

Fig. 18. Analytical model of the improved method: (a) negative resistor connected in parallel and (b) negative resistor connected in series.

Fig. 19. Simulation results of agreement evaluation index for $\beta_{i}=0.0003$ using two-peak method with improvement: (a) negative resistor connected in parallel with $\bar{\zeta}_{\mathrm{np} i}=-\bar{\delta}_{p i}+0.02$ and (b) negative resistor connected in series with $\bar{\zeta}_{\mathrm{ns} i}=-\bar{\delta}_{p i}+0.02$.

Fig. 20. Simulated magnitude plots of nondimensional compliance for $\beta_{i}=0.0003$ : (a) $\bar{\gamma}_{L i}=0$ and $\bar{\delta}_{p i}=3$; (b) $\bar{\gamma}_{L i}=0, \bar{\delta}_{p i}=3$, and $\bar{\zeta}_{\mathrm{npi}}=-2.98$; (c) $\bar{\gamma}_{L i}=0, \bar{\delta}_{p i}=3$, and $\bar{\zeta}_{\mathrm{ns} i}=-2.98$; (d) $\bar{\gamma}_{L i}=1$ and $\bar{\delta}_{p i}=3$; (e) $\bar{\gamma}_{L i}=1, \bar{\delta}_{p i}=3$, and $\bar{\zeta}_{\mathrm{np} i}=-2.98$; and (f) $\bar{\gamma}_{L i}=1, \bar{\delta}_{p i}=3$, and $\bar{\zeta}_{\mathrm{ns} i}=-2.98$.

Fig. 21. Schematic of experimental apparatus.

Fig. 22. Schematic diagram of negative resistors realized using negative impedance converter: (a) negative resistor for parallel connection and (b) negative resistor for series connection.

Fig. 23. Calculation model for the cantilever with piezoelectric elements to derive the mode shapes. 
Fig. 24. Experimental results of nondimensional compliance when the electrodes of piezoelectric element A are short- and open-circuited: (a) first vibration mode, (b) second vibration mode, (c) third vibration mode, (d) fourth vibration mode, and (e) fifth vibration mode.

Fig. 25. Experimental results of nondimensional compliance when the inductor was coupled to piezoelectric element A: (a) first vibration mode, (b) second vibration mode, (c) third vibration mode, (d) fourth vibration mode, and (e) fifth vibration mode.

Fig. 26. Experimental results of nondimensional compliance of piezoelectric element A when the two-peak method with a negative resistor was applied: (a) first vibration mode, (b) second vibration mode, (c) third vibration mode, (d) fourth vibration mode, and (e) fifth vibration mode.

Fig. 27. Experimental results of the nondimensional compliance of piezoelectric element $A$ using inductor and additional resistor. 


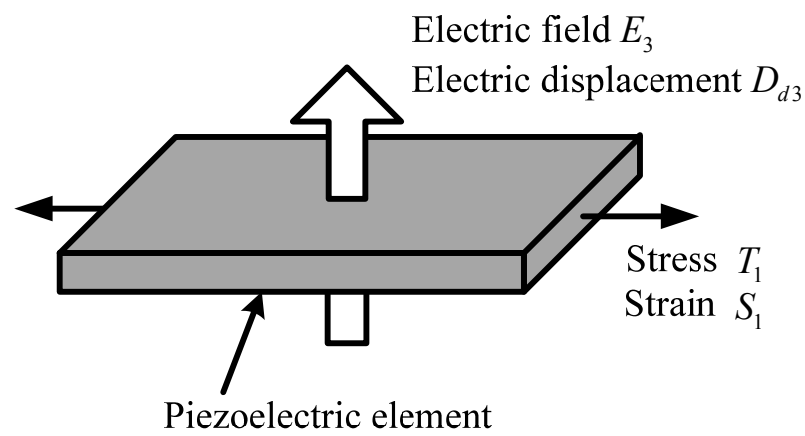

(a)

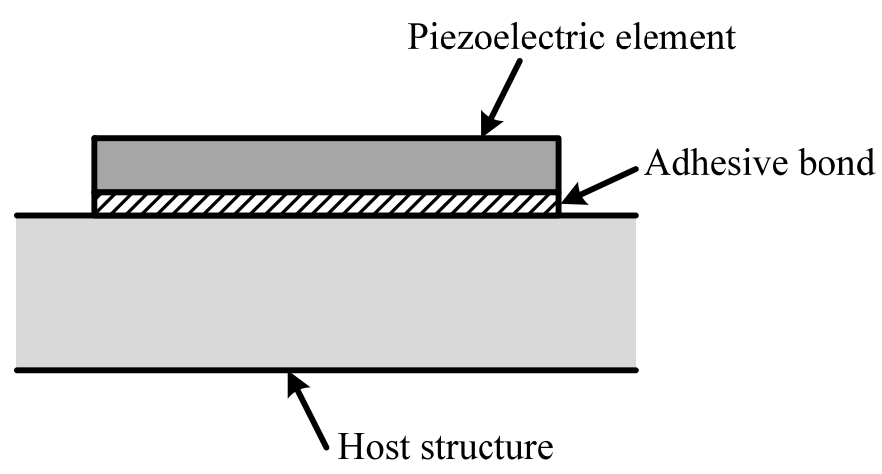

(b)

Fig. 1. Schematic diagrams of plate-type piezoelectric element: (a) plate-type piezoelectric element and (b) piezoelectric element attached to host structure by adhesive bond.

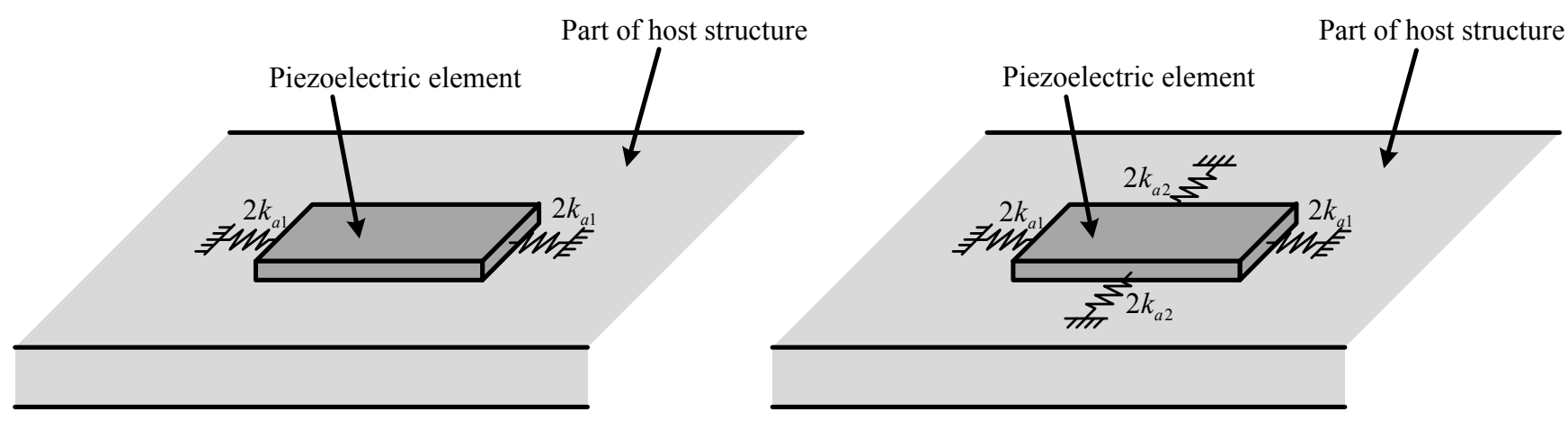

(a)

(b)

Fig. 2. Analytical model of the stiffness of the bonding layer using equivalent springs: (a) one-dimensional model and (b) two-dimensional model.

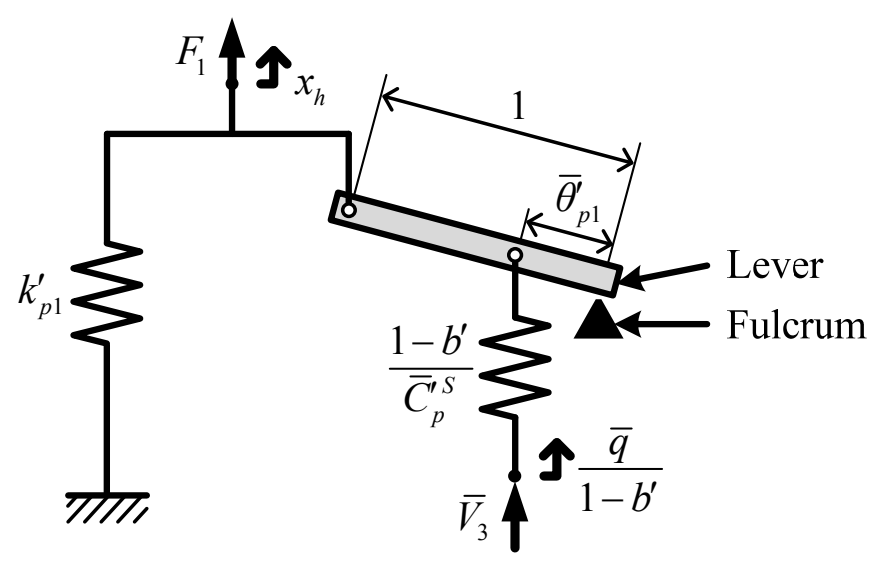

(a)

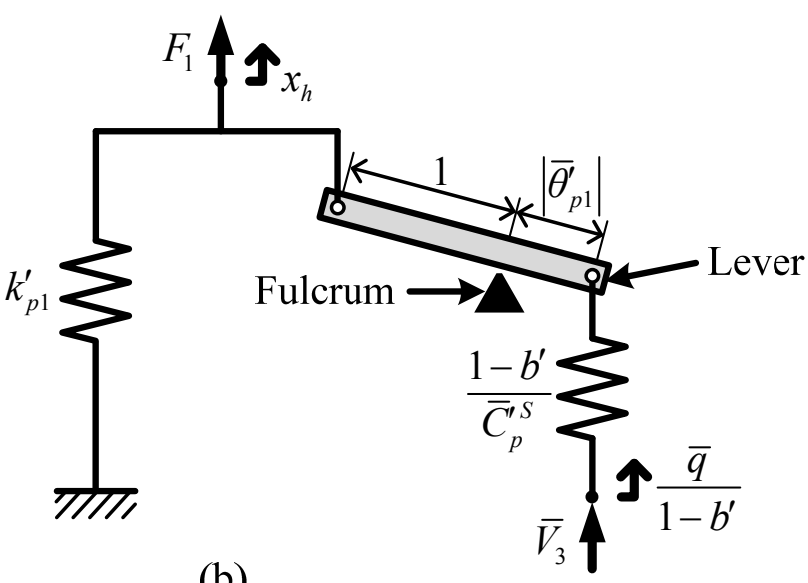

(b)

Fig. 3. Equivalent mechanical model of piezoelectric element and its lever: (a) $\bar{\theta}_{p 1}^{\prime} \geq 0$ and (b) $\bar{\theta}_{p 1}^{\prime}<0$. 


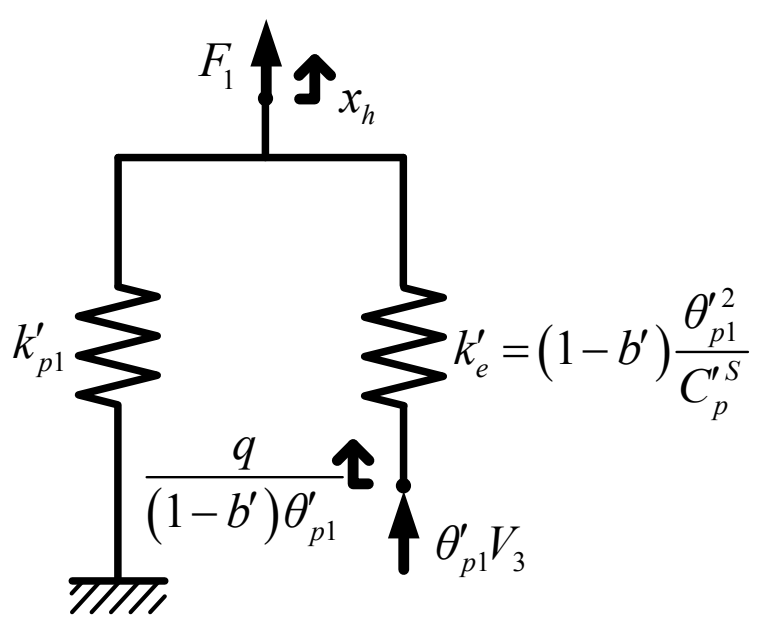

Fig. 4. Equivalent mechanical model without levers.

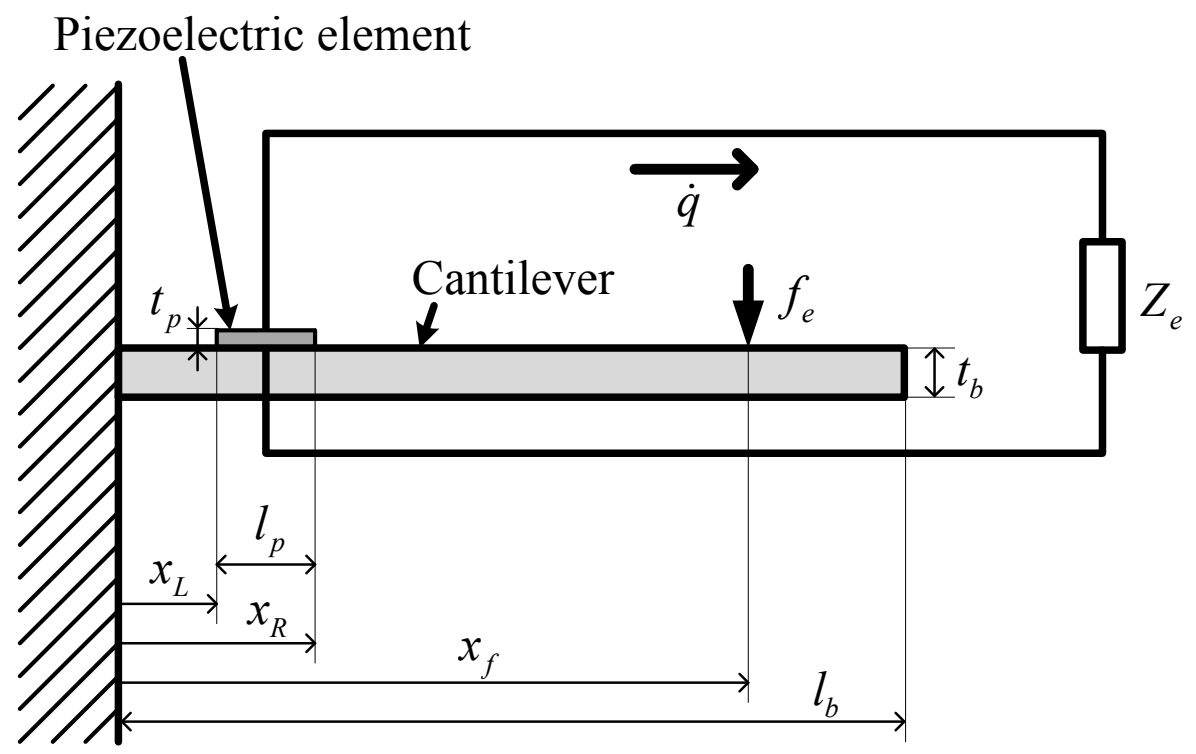

Fig. 5. Analytical model of passive vibration suppression system using piezoelectric element and electrical impedance. 


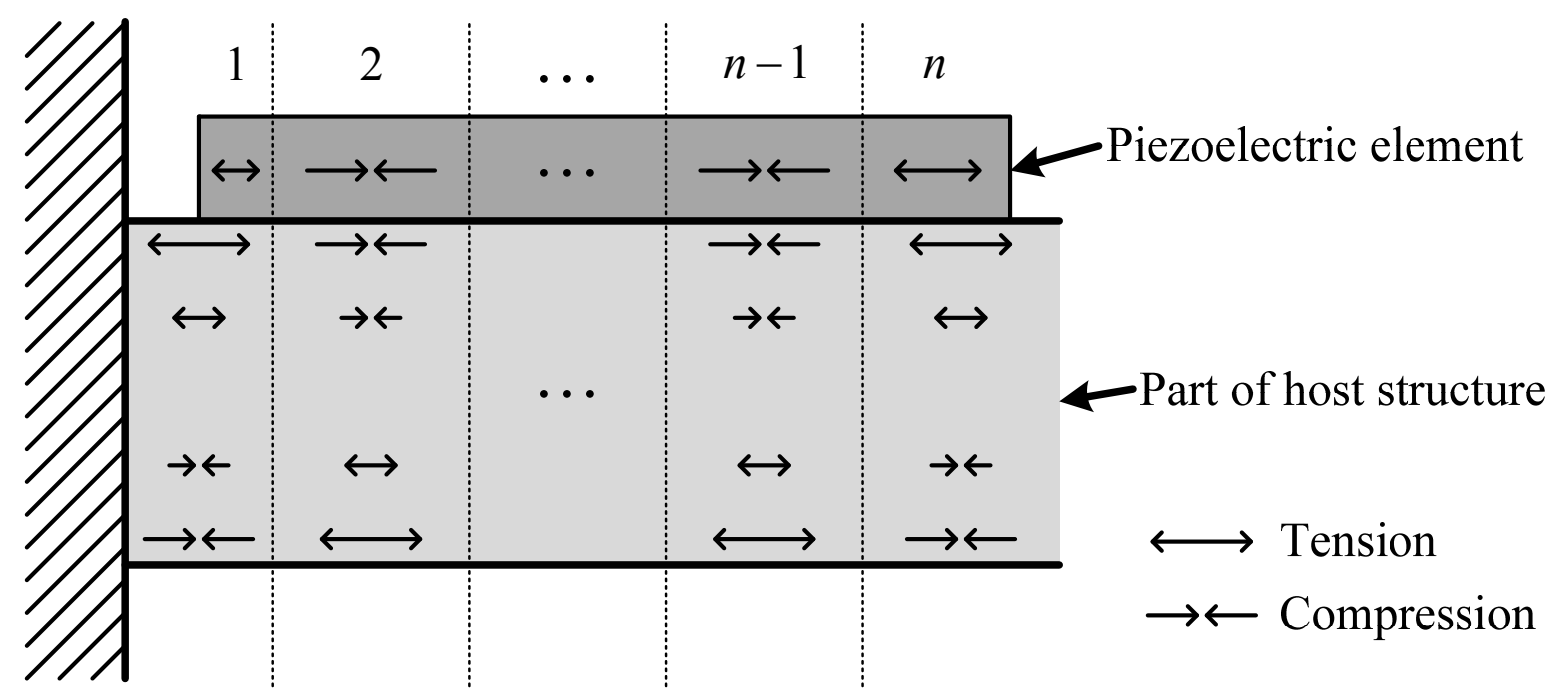

Fig. 6. Conceptual diagram of complex deformation of piezoelectric element.

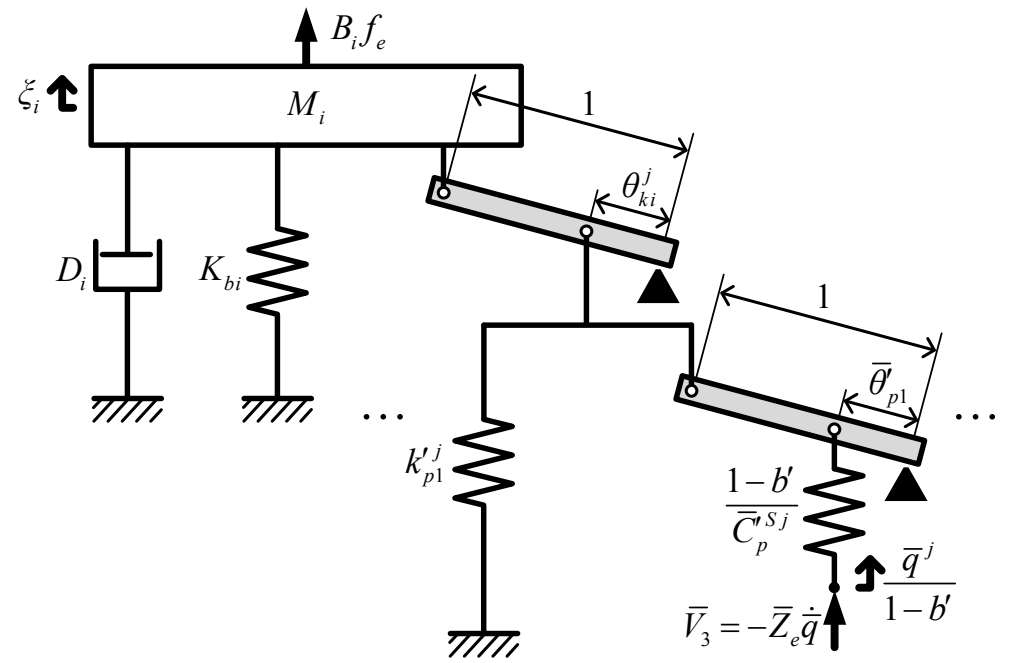

(a)

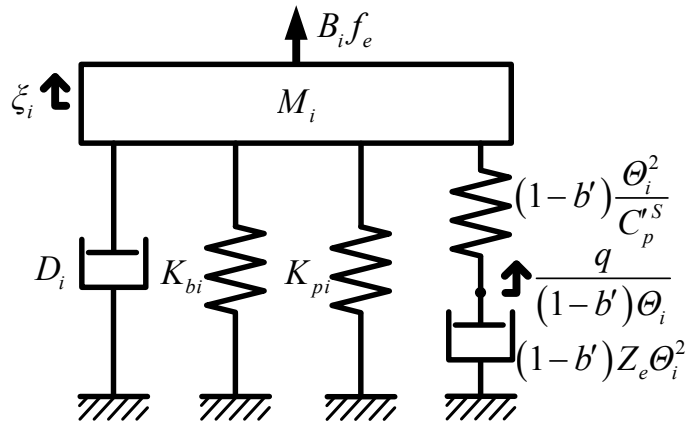

(b)

Fig. 7. Equivalent mechanical models for targeted vibration mode when the piezoelectric element is shunted with electrical impedance: (a) equivalent model with levers of $\theta_{k i}^{j}>0$ and $\theta_{p 1}^{\prime}>0$ and (b) equivalent model without virtual levers.

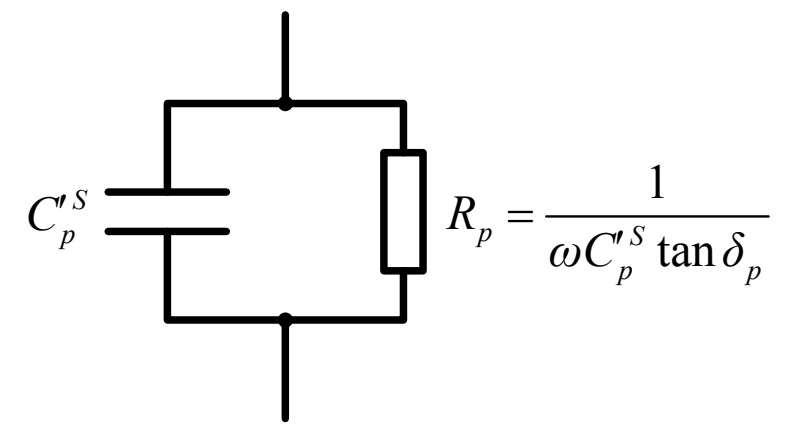

Fig. 8. Equivalent circuit of piezoelectric element with dielectric loss. 


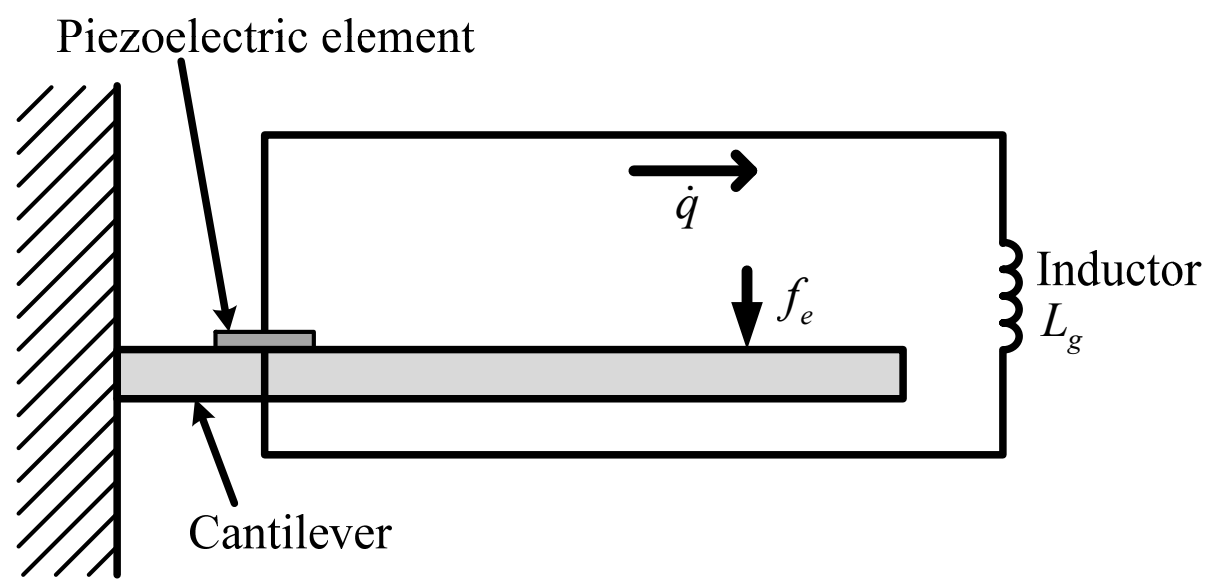

Fig. 9. Analytical model of two-peak method.

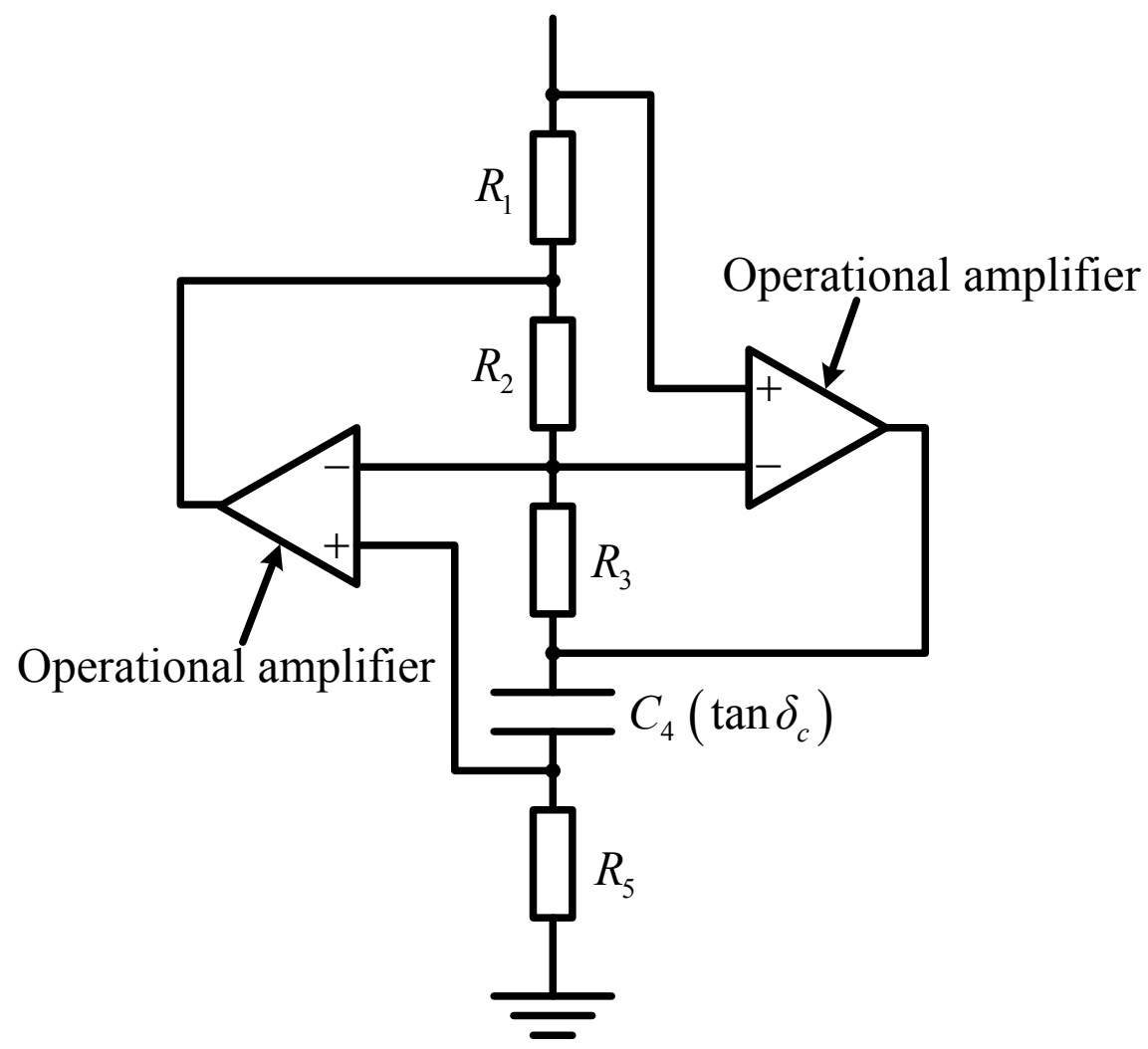

(a)

(b)

Fig. 10. Schematic diagram of the simulated inductor using generalized impedance converter and its equivalent circuit: (a) simulated inductor circuit and (b) equivalent circuit. 


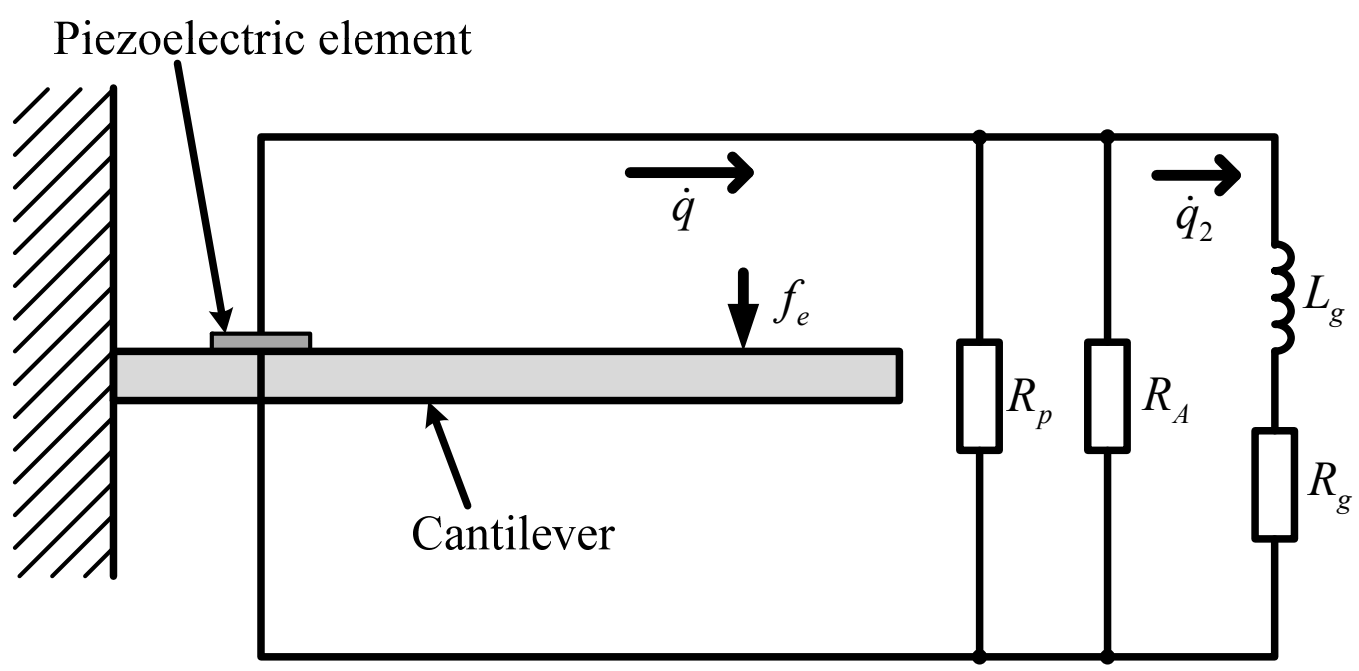

Fig. 11. Analytical model of two-point intersection method.
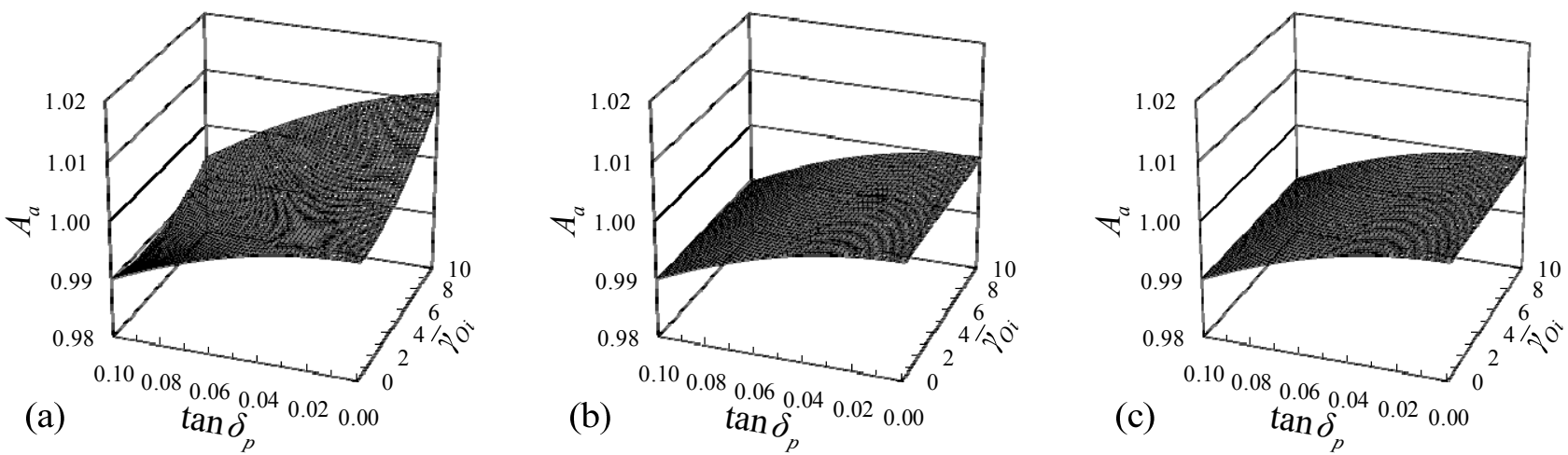

Fig. 12. Simulation results of the agreement evaluation index for conventional method using different values of $\bar{\gamma}_{O i}$ and $\tan \delta_{p}$ : (a) $\beta_{i}=0.03$, (b) $\beta_{i}=0.003$, and (c) $\beta_{i}=0.0003$. 

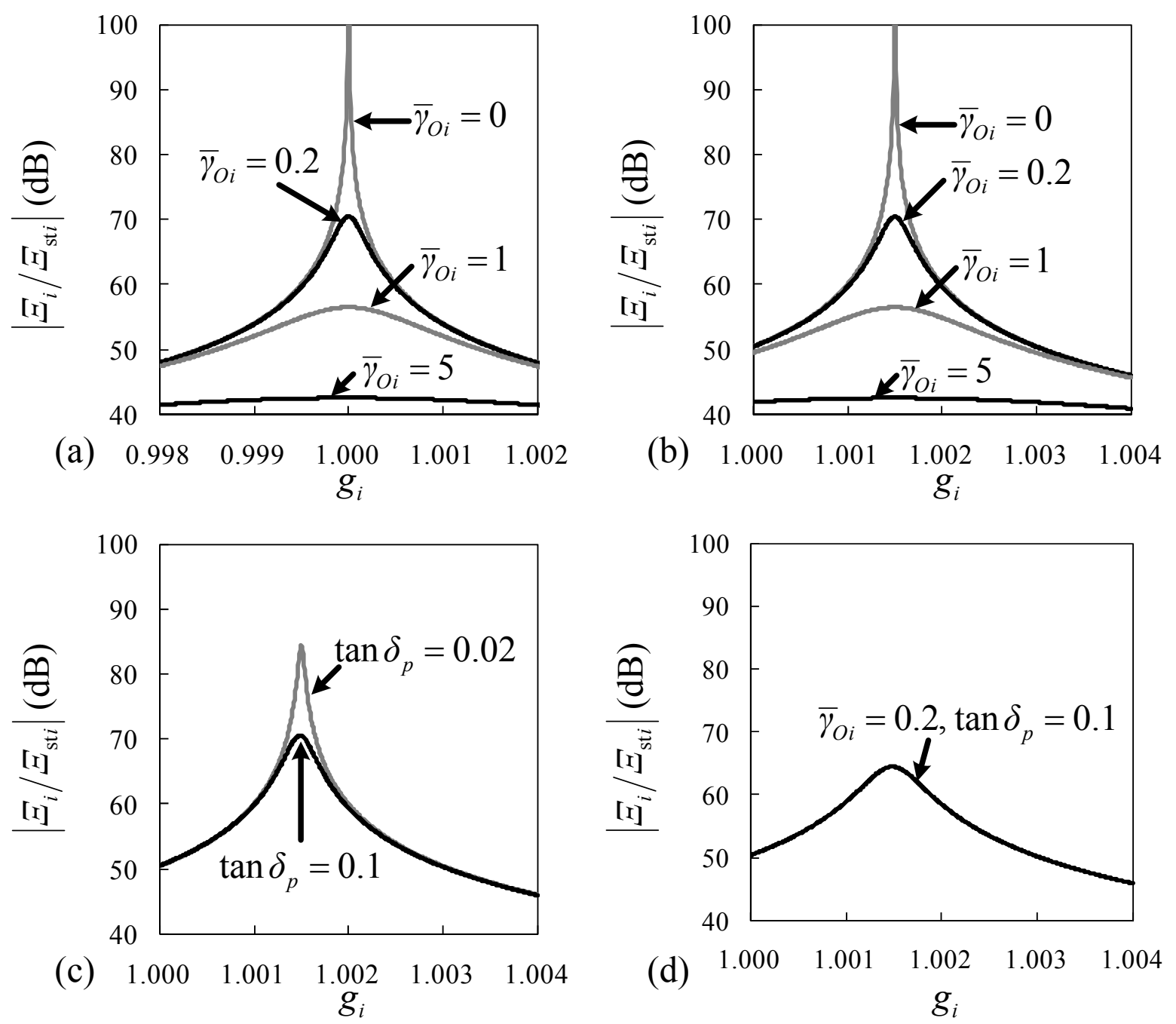

Fig. 13. Simulated magnitude plots of nondimensional compliance using $\beta_{i}=0.003$ : (a) short-circuited electrodes with varying $\bar{\gamma}_{O i}$, (b) open-circuited electrodes with $\tan \delta_{p}=0$ and varying $\bar{\gamma}_{O i}$, (c) open-circuited electrodes with $\bar{\gamma}_{O i}=0$ and varying $\tan \delta_{p}$, and (d) open-circuited electrodes with $\bar{\gamma}_{O i}=0.2$ and $\tan \delta_{p}=0.1$.
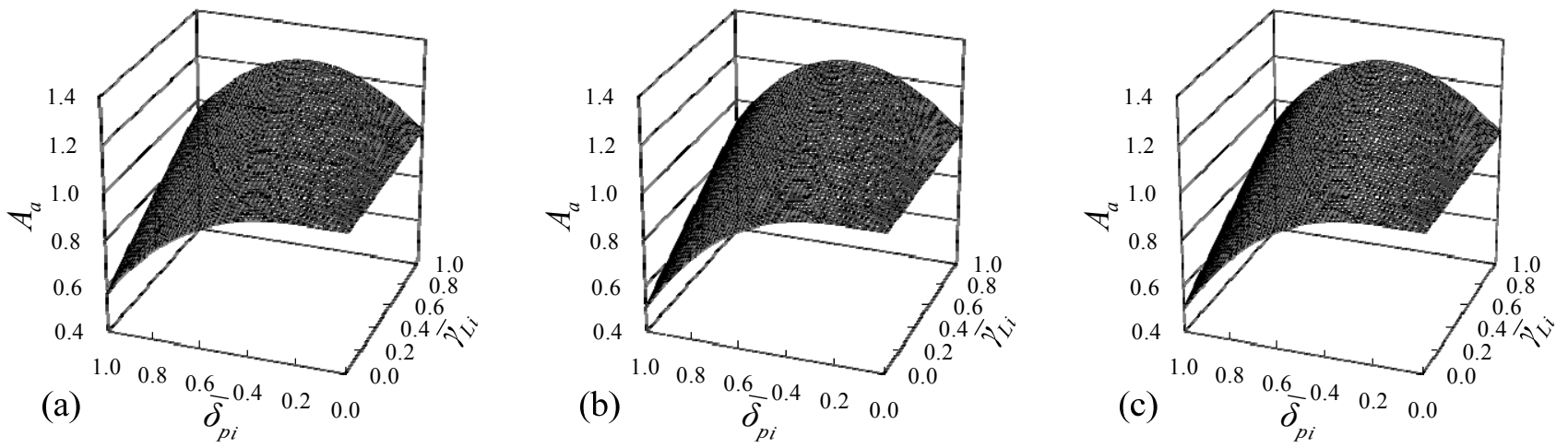

Fig. 14. Simulation results of the agreement evaluation index for two-peak method using different values of $\bar{\gamma}_{L i}$ and $\bar{\delta}_{p i}$ : (a) $\beta_{i}=0.03$, (b) $\beta_{i}=0.003$, and (c) $\beta_{i}=0.0003$. 

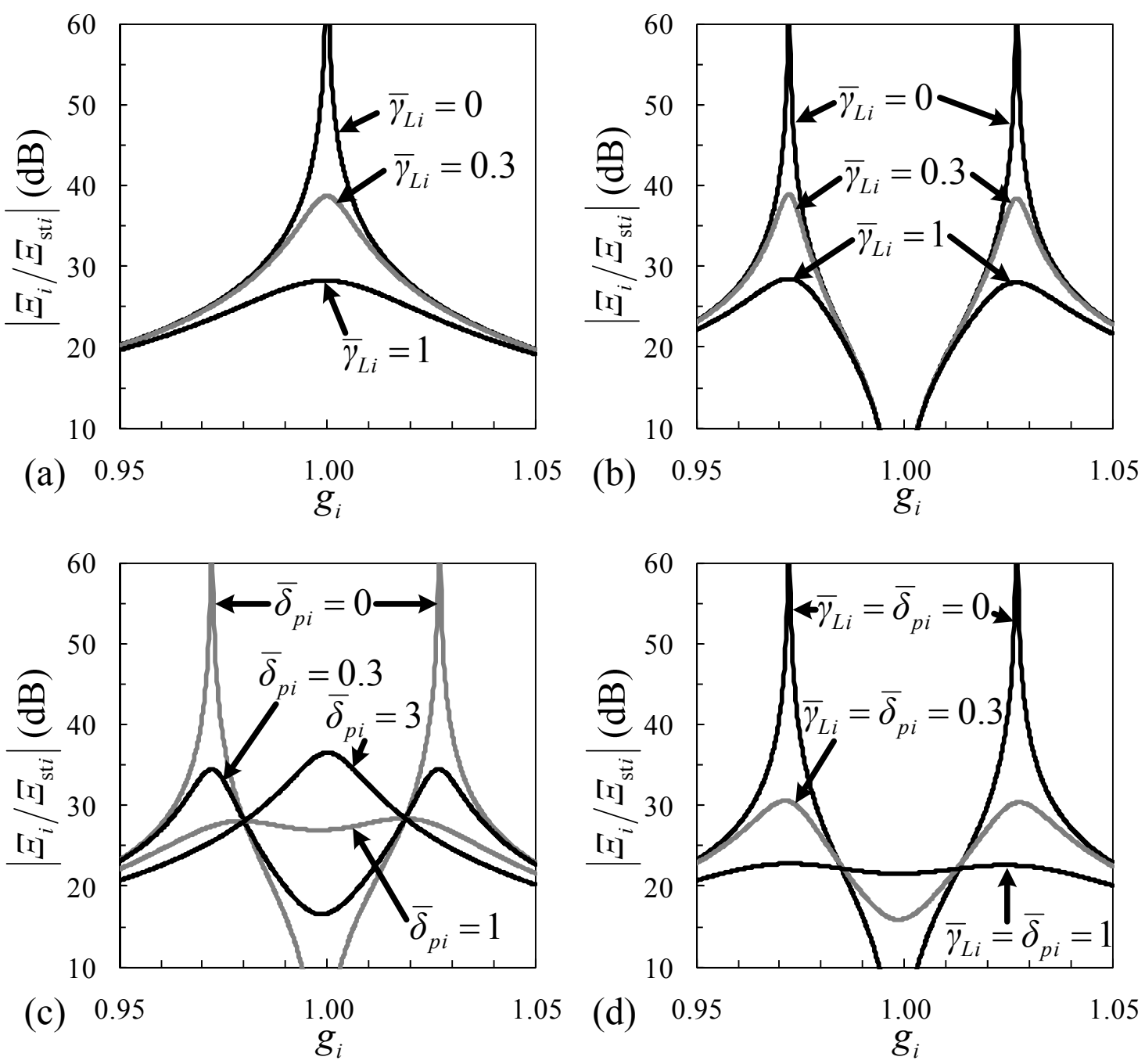

Fig. 15. Simulated magnitude plots of nondimensional compliance using $\beta_{i}=0.003$ : (a) short-circuited electrodes with varying $\bar{\gamma}_{L i}$, (b) inductive circuit with $\bar{\delta}_{p i}=0$ and varying $\bar{\gamma}_{L i}$, (c) inductive circuit with $\bar{\gamma}_{L i}=0$ and varying $\bar{\delta}_{p i}$, and (d) inductive circuit with various combinations of $\bar{\gamma}_{L i}$ and $\bar{\delta}_{p i}$.
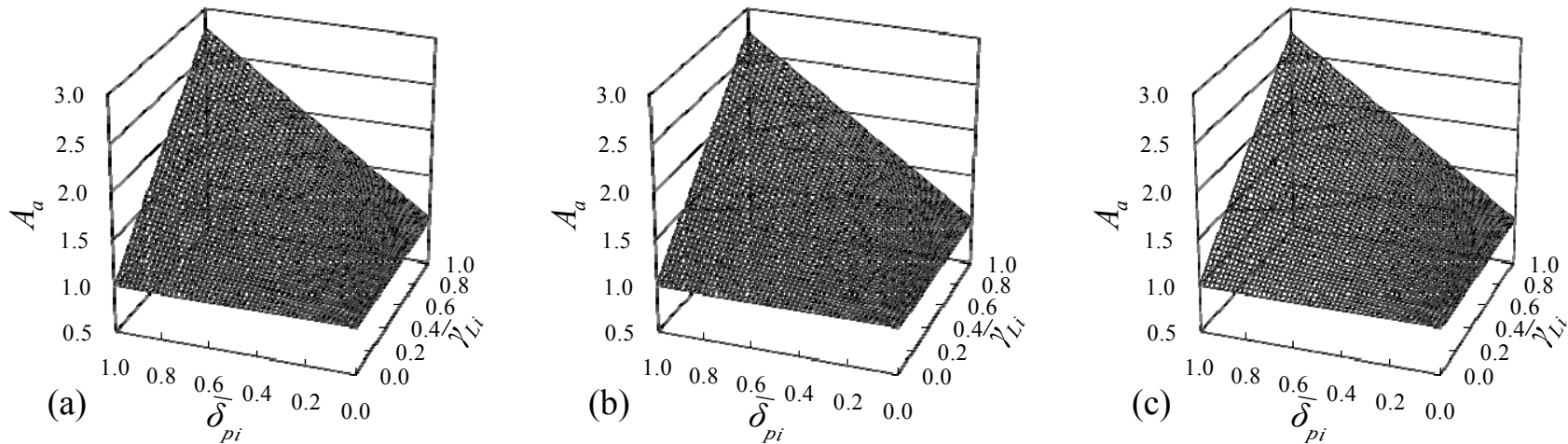

Fig. 16. Simulation results of agreement evaluation index for two-point intersection method using different values of $\bar{\gamma}_{L i}$ and $\bar{\delta}_{p i}:$ (a) $\beta_{i}=0.03$, (b) $\beta_{i}=0.003$, and (c) $\beta_{i}=0.0003$. 

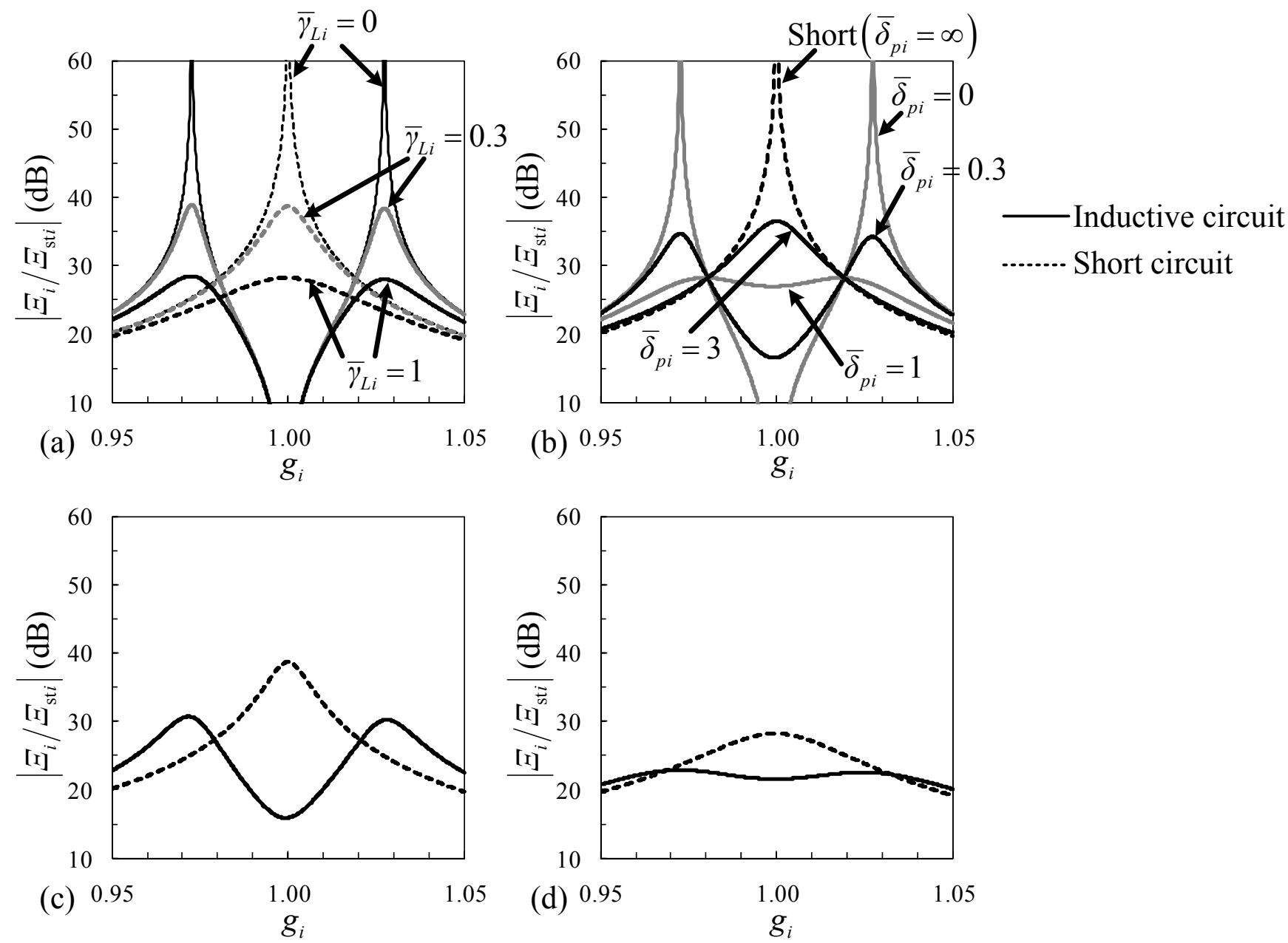

Fig. 17. Simulated magnitude plots of nondimensional compliance for $\beta_{i}=0.003$ : (a) $\bar{\delta}_{p i}=0$ and varying $\bar{\gamma}_{L i}$, (b) $\bar{\gamma}_{L i}=0$ and varying $\bar{\delta}_{p i}$, (c) $\bar{\gamma}_{L i}=\bar{\delta}_{p i}=0.3$, and (d) $\bar{\gamma}_{L i}=\bar{\delta}_{p i}=1$.

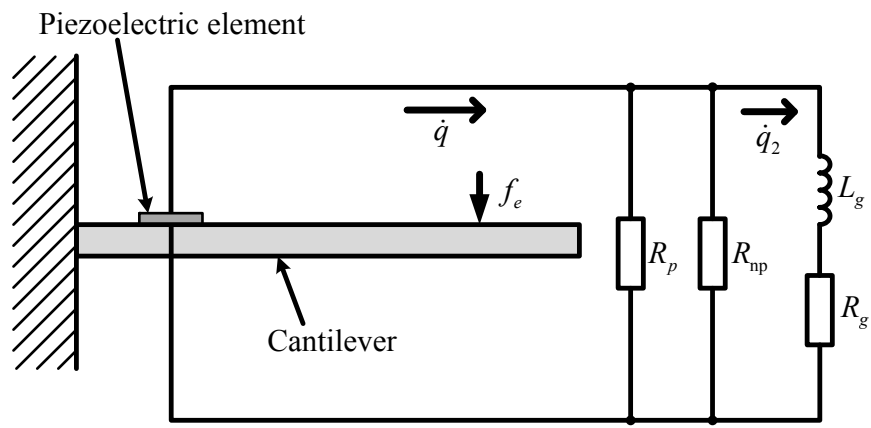

(a)

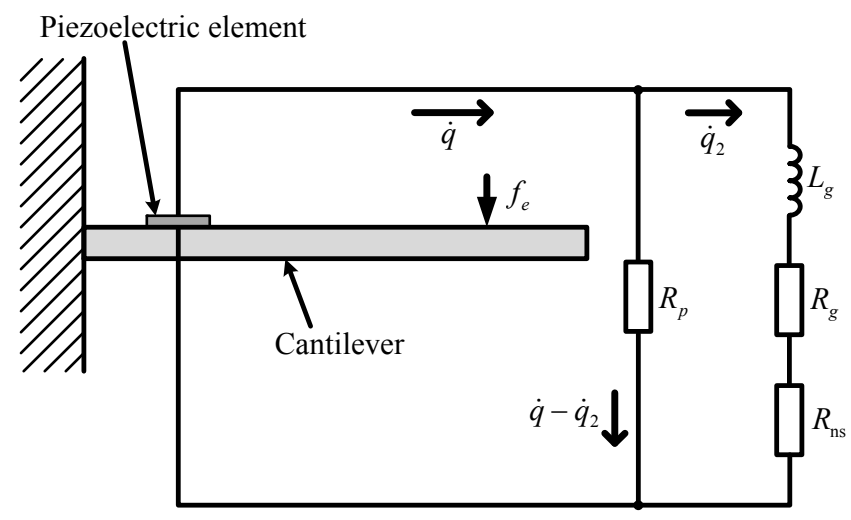

(b)

Fig. 18. Analytical model of the improved method: (a) negative resistor connected in parallel and (b) negative resistor connected in series. 

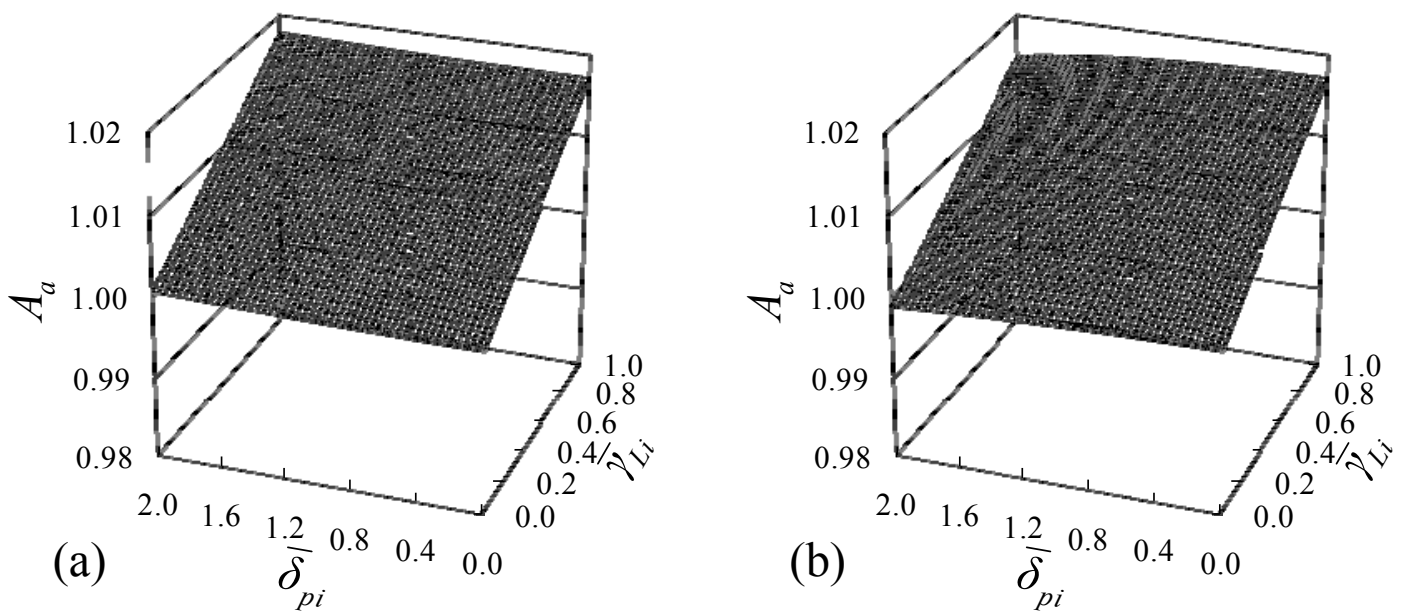

Fig. 19. Simulation results of agreement evaluation index for $\beta_{i}=0.0003$ using two-peak method with improvement: (a) negative resistor connected in parallel with $\bar{\zeta}_{\mathrm{np} i}=-\bar{\delta}_{p i}+0.02$ and (b) negative resistor connected in series with $\bar{\zeta}_{\mathrm{ns} i}=-\bar{\delta}_{p i}+0.02$.
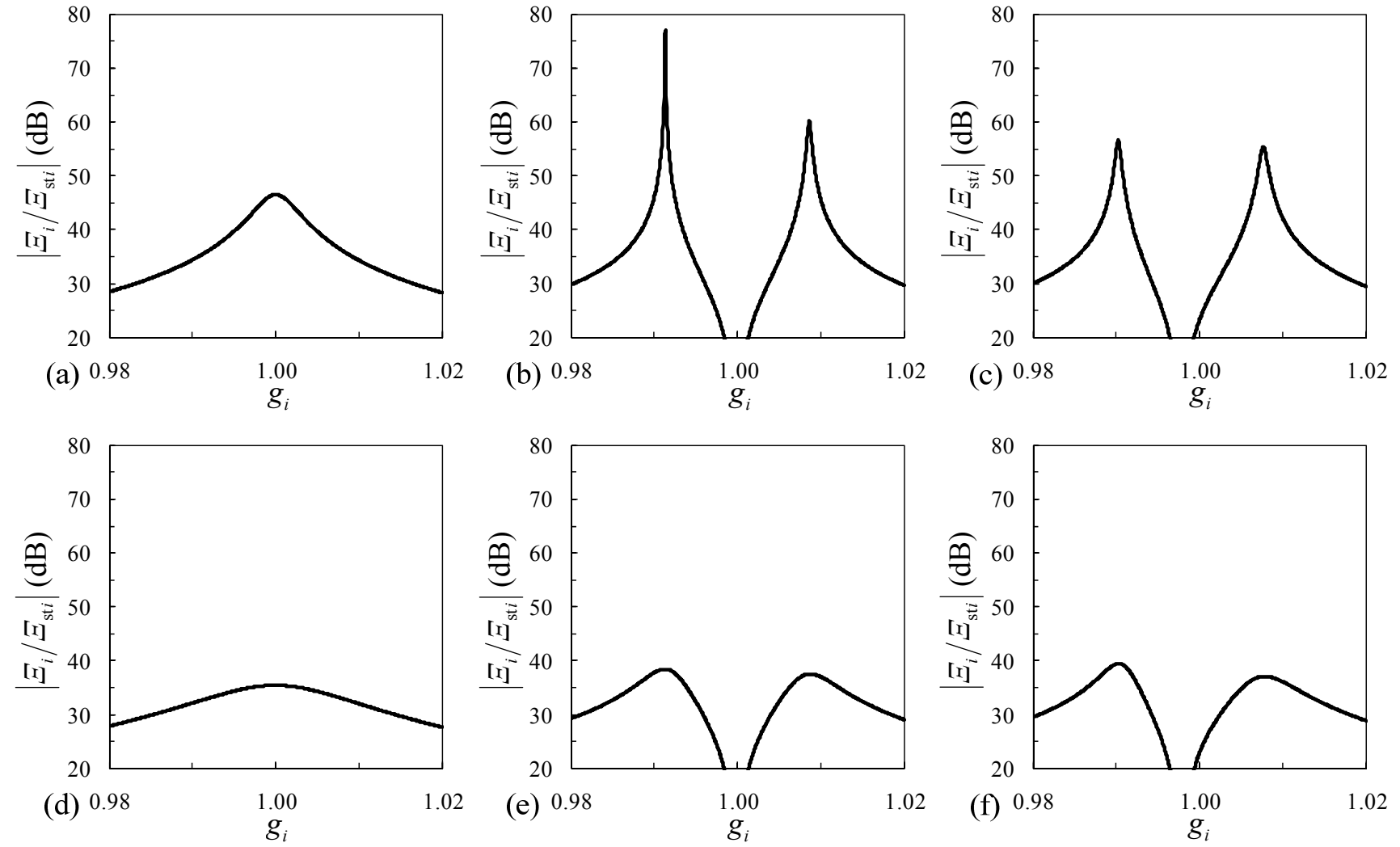

Fig. 20. Simulated magnitude plots of nondimensional compliance for $\beta_{i}=0.0003:$ (a) $\bar{\gamma}_{L i}=0$ and $\bar{\delta}_{p i}=3$; (b) $\bar{\gamma}_{L i}=0, \bar{\delta}_{p i}=3$, and $\bar{\zeta}_{\mathrm{np} i}=-2.98$; (c) $\bar{\gamma}_{L i}=0, \bar{\delta}_{p i}=3$, and $\bar{\zeta}_{\mathrm{ns} i}=-2.98$; (d) $\bar{\gamma}_{L i}=1$ and $\bar{\delta}_{p i}=3$; (e) $\bar{\gamma}_{L i}=1, \bar{\delta}_{p i}=3$, and $\bar{\zeta}_{\mathrm{np} i}=-2.98$; and (f) $\bar{\gamma}_{L i}=1, \bar{\delta}_{p i}=3$, and $\bar{\zeta}_{\mathrm{ns} i}=-2.98$. 


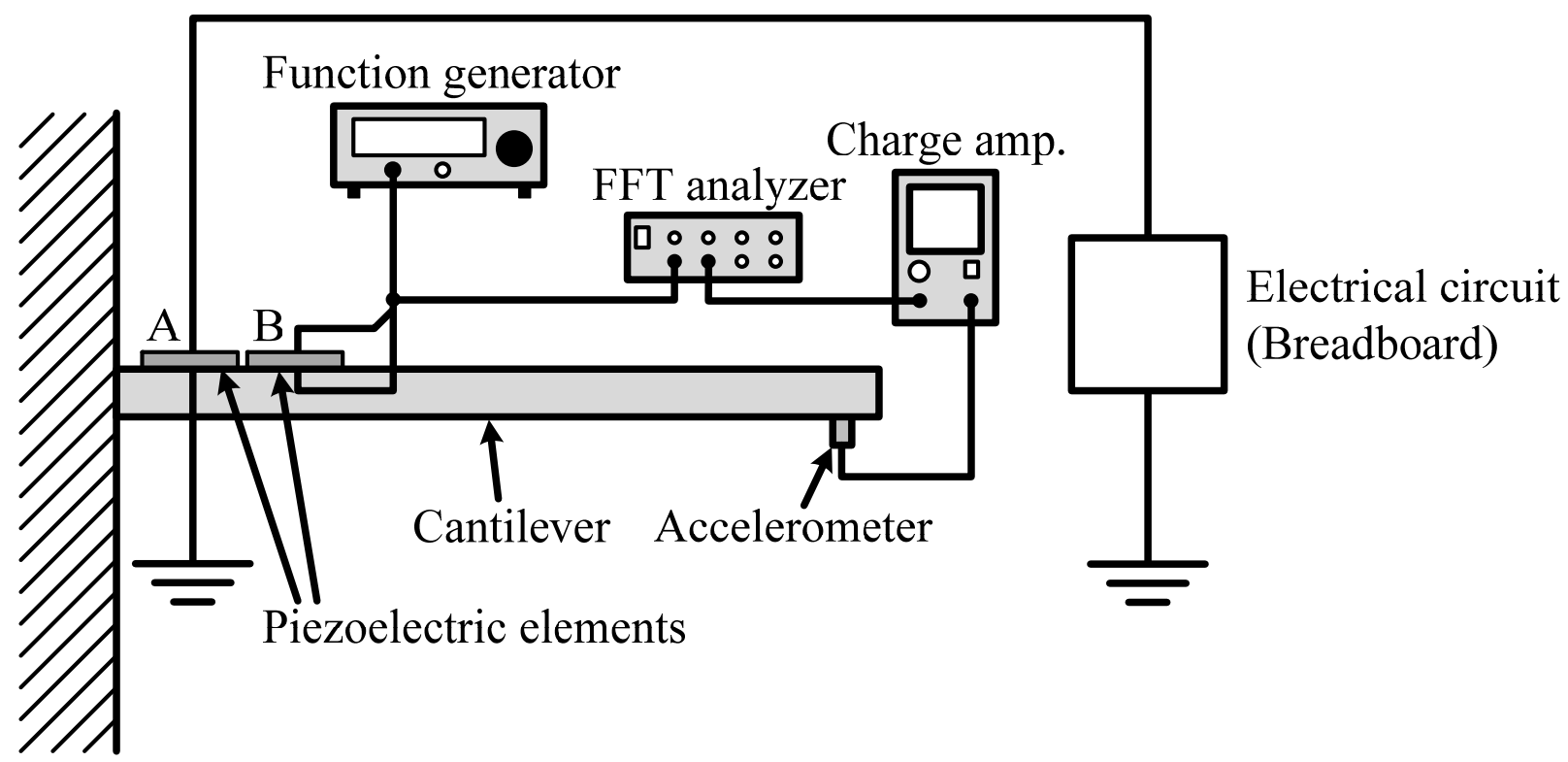

Fig. 21. Schematic of experimental apparatus.

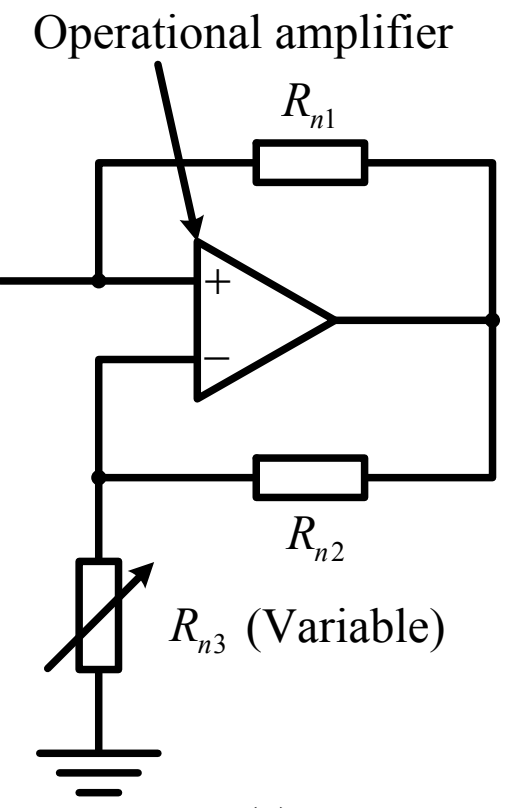

(a)

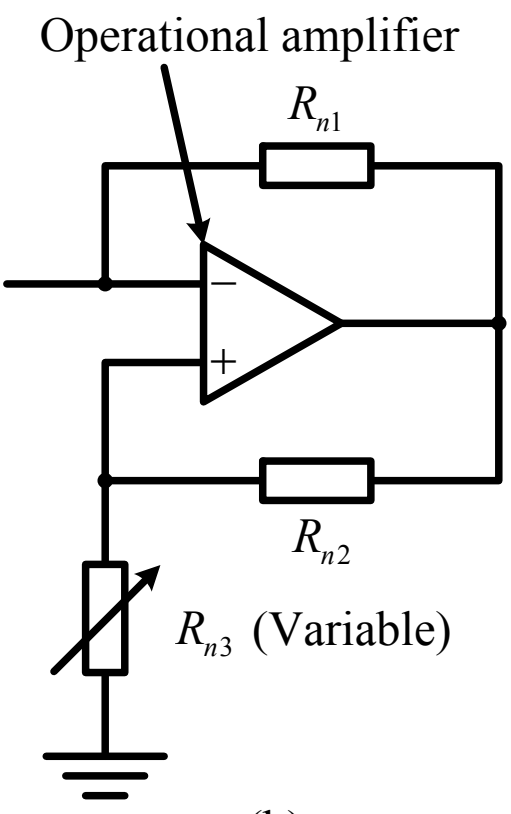

(b)

Fig. 22. Schematic diagram of negative resistors realized using negative impedance converter: (a) negative resistor for parallel connection and (b) negative resistor for series connection. 


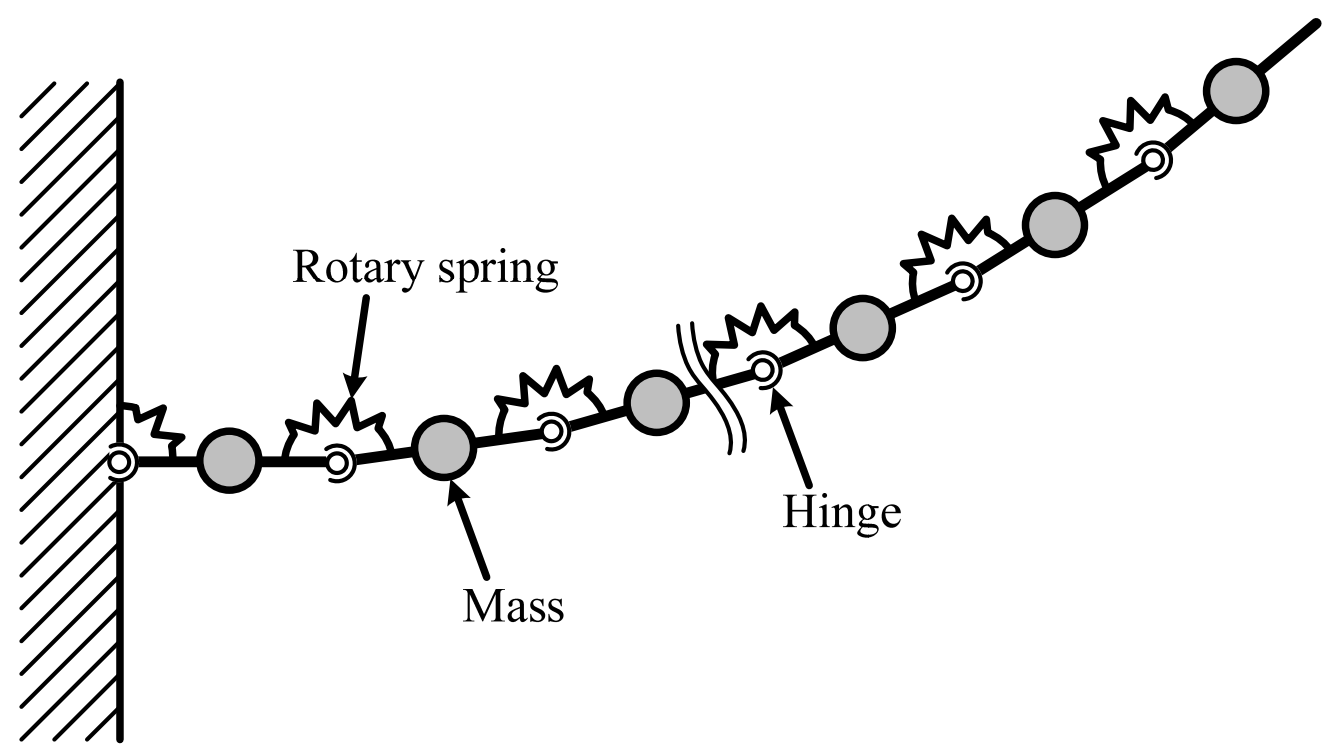

Fig. 23. Calculation model for the cantilever with piezoelectric elements to derive the mode shapes.
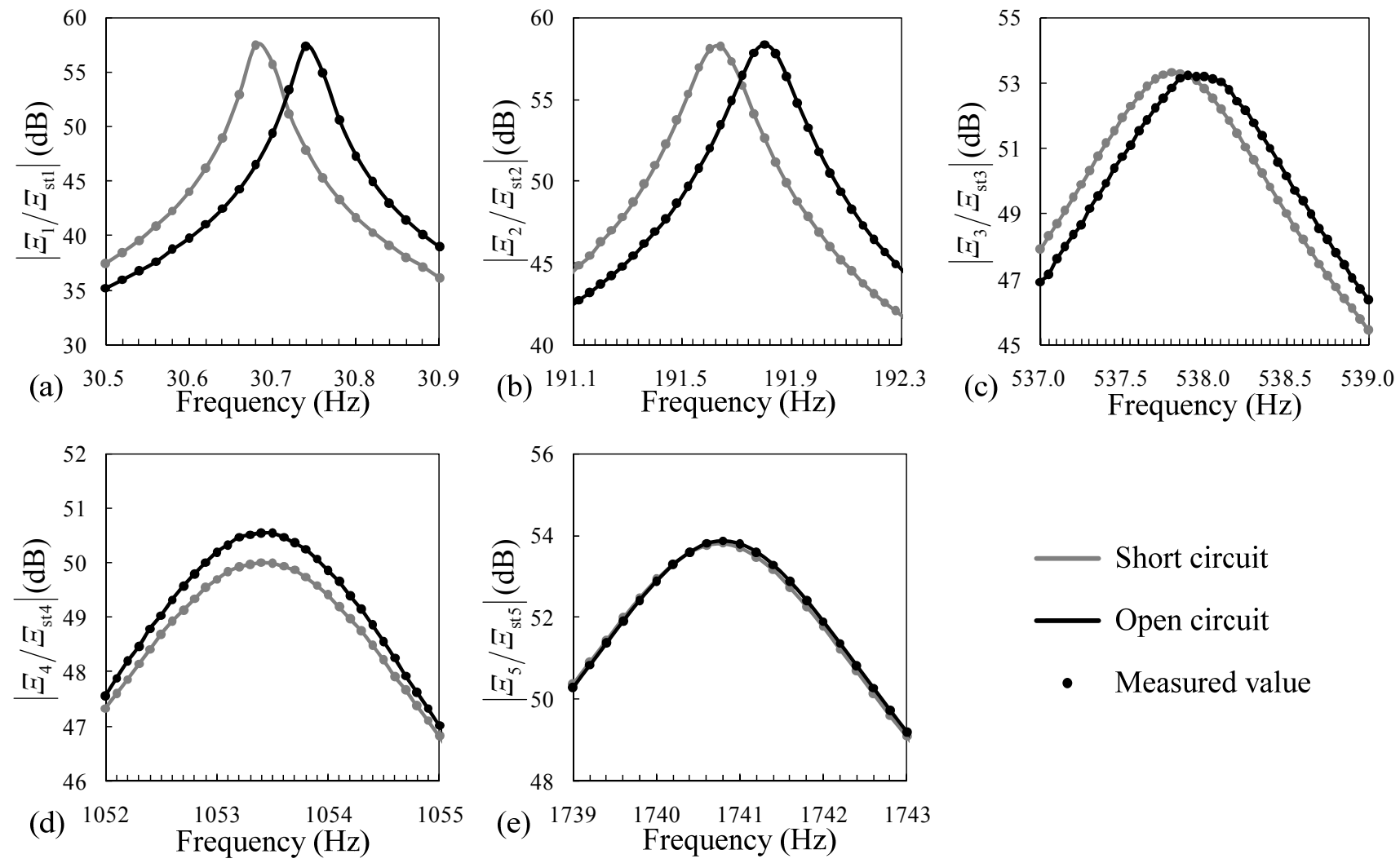

- Short circuit

- Open circuit

- Measured value

Fig. 24. Experimental results of nondimensional compliance when the electrodes of piezoelectric element $A$ are short- and open-circuited: (a) first vibration mode, (b) second vibration mode, (c) third vibration mode, (d) fourth vibration mode, and (e) fifth vibration mode. 

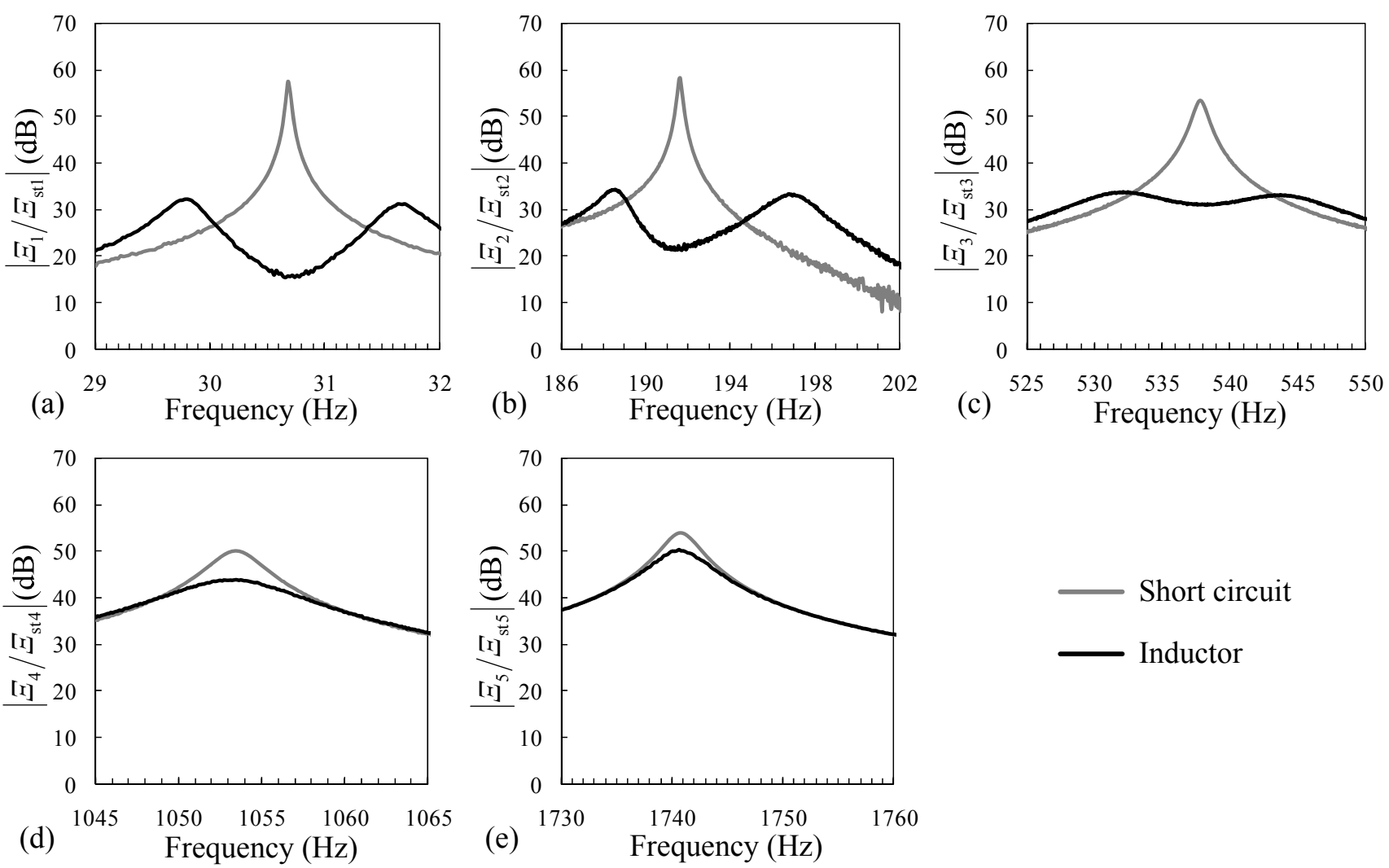

Fig. 25. Experimental results of nondimensional compliance when the inductor was coupled to piezoelectric element A: (a) first vibration mode, (b) second vibration mode, (c) third vibration mode, (d) fourth vibration mode, and (e) fifth vibration mode. 

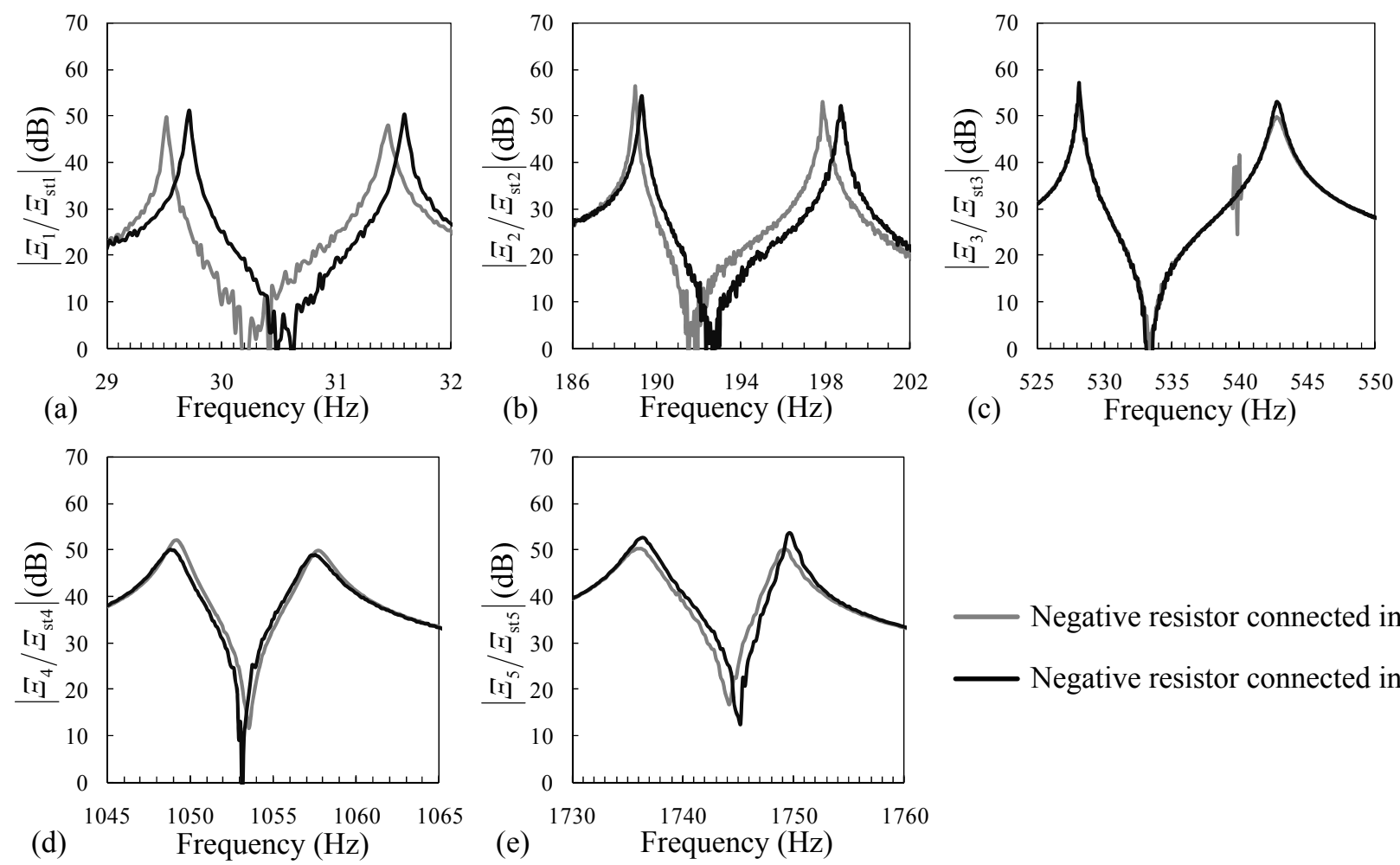

Negative resistor connected in series

Negative resistor connected in parallel

Fig. 26. Experimental results of nondimensional compliance of piezoelectric element A when the two-peak method with a negative resistor was applied: (a) first vibration mode, (b) second vibration mode, (c) third vibration mode, (d) fourth vibration mode, and (e) fifth vibration mode.

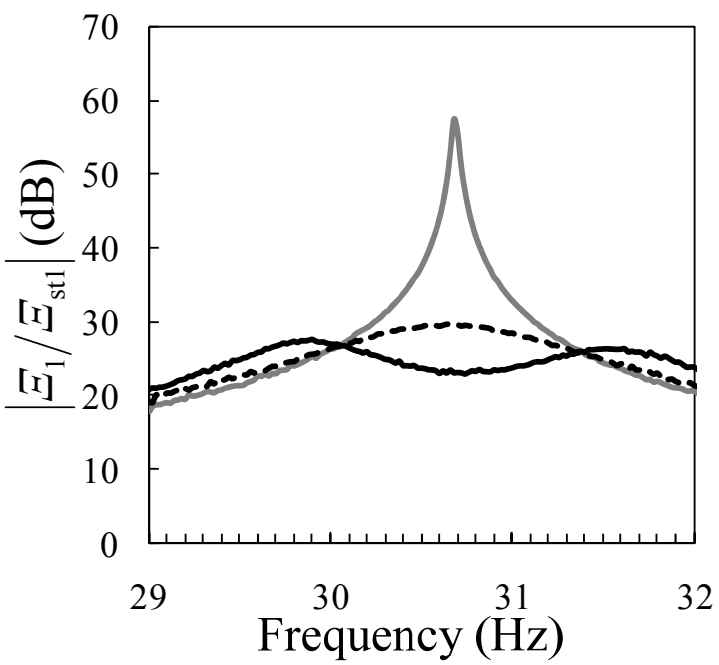

- Short circuit

$-R_{\mathrm{ap}}=3.0 \times 10^{6}(\Omega)$

$\cdots-R_{\text {ap }}=1.0 \times 10^{6}(\Omega)$

Fig. 27. Experimental results of the nondimensional compliance of piezoelectric element A using inductor and additional resistor. 